\title{
HAT-P-57b: A SHORT-PERIOD GIANT PLANET TRANSITING A BRIGHT RAPIDLY ROTATING A8V STAR CONFIRMED VIA DOPPLER TOMOGRAPHY*
}

\author{
J. D. Hartman ${ }^{1}$, G. Á. Bakos ${ }^{1,12,13}$, L. A. Buchhave ${ }^{2,3}$, G. Torres ${ }^{2}$, D. W. Latham ${ }^{2}$, G. Kovács ${ }^{4}$, W. Bhatti ${ }^{1}$, Z. Csubry ${ }^{1}$, \\ M. de Val-Borro ${ }^{1}$, K. Penev ${ }^{1}$, C. X. Huang ${ }^{1}$, B. Béky ${ }^{5}$, A. Bieryla ${ }^{2}$, S. N. Quinn ${ }^{6}$, A. W. Howard ${ }^{7}$, G. W. Marcy ${ }^{8}$, \\ J. A. Johnson ${ }^{2}$, H. IsaAcson ${ }^{8}$, D. A. Fischer ${ }^{9}$, R. W. Noyes ${ }^{2}$, E. FAlCo ${ }^{2}$, G. A. Esquerdo ${ }^{2}$, R. P. KNOx ${ }^{10}$, P. Hinz ${ }^{10}$, J. LÁzÁr ${ }^{11}$, \\ I. PAPP ${ }^{11}$, AND P. SÁRI ${ }^{11}$ \\ ${ }^{1}$ Department of Astrophysical Sciences, Princeton University, Princeton, NJ 08544, USA; jhartman@astro.princeton.edu \\ ${ }^{2}$ Harvard-Smithsonian Center for Astrophysics, Cambridge, MA 02138, USA \\ ${ }^{3}$ Centre for Star and Planet Formation, Natural History Museum of Denmark, University of Copenhagen, DK-1350 Copenhagen, Denmark \\ ${ }^{4}$ Konkoly Observatory, Budapest, Hungary \\ ${ }^{5}$ Google, USA \\ ${ }^{6}$ Department of Physics and Astronomy, Georgia State University, Atlanta, GA 30303, USA \\ ${ }^{7}$ Institute for Astronomy, University of Hawaii, Honolulu, HI 96822, USA \\ 8 Department of Astronomy, University of California, Berkeley, CA, USA \\ 9 Department of Astronomy, Yale University, New Haven, CT, USA \\ ${ }^{10}$ Steward Observatory, University of Arizona, 933 N. Cherry Ave., Tucson, AZ 85721, USA \\ ${ }^{11}$ Hungarian Astronomical Association, Budapest, Hungary \\ Received 2015 September 16; accepted 2015 October 29; published 2015 December 11
}

\begin{abstract}
We present the discovery of HAT-P-57b, a $P=2.4653$ day transiting planet around a $V=10.465 \pm 0.029$ mag, $\quad T_{\text {eff }}=7500 \pm 250 \mathrm{~K}$ main sequence A8V star with a projected rotation velocity of $v \sin i=102.1 \pm 1.3 \mathrm{~km} \mathrm{~s}^{-1}$. We measure the radius of the planet to be $R=1.413 \pm 0.054 R_{\mathrm{J}}$ and, based on RV observations, place a $95 \%$ confidence upper limit on its mass of $M<1.85 M_{\mathrm{J}}$. Based on theoretical stellar evolution models, the host star has a mass and radius of $1.47 \pm 0.12 M_{\odot}$ and $1.500 \pm 0.050 R_{\odot}$, respectively. Spectroscopic observations made with Keck-I/HIRES during a partial transit event show the Doppler shadow of HAT-P-57b moving across the average spectral line profile of HAT-P-57, confirming the object as a planetary system. We use these observations, together with analytic formulae that we derive for the line profile distortions, to determine the projected angle between the spin axis of HAT-P-57 and the orbital axis of HAT-P-57b. The data permit two possible solutions, with $-16.7<\lambda<3.3$ or $27^{\circ} .6<\lambda<57^{\circ} .4$ at $95 \%$ confidence, and with relative probabilities for the two modes of $26 \%$ and $74 \%$, respectively. Adaptive optics imaging with MMT/Clio2 reveals an object located 2"7 from HAT-P-57 consisting of two point sources separated in turn from each other by $0 .{ }^{\prime \prime} 22$. The $H$ - and $L^{\prime}$-band magnitudes of the companion stars are consistent with their being physically associated with HAT-P-57, in which case they are stars of mass $0.61 \pm 0.10 M_{\odot}$ and $0.53 \pm 0.08 M_{\odot}$. HAT-P-57 is the most rapidly rotating star, and only the fourth main sequence A star, known to host a transiting planet.
\end{abstract}

Key words: planetary systems - stars: individual (HAT-P-57) - techniques: photometric - techniques: spectroscopic

Supporting material: machine-readable and VO table

\section{INTRODUCTION}

In order to develop a comprehensive understanding of exoplanetary systems it is necessary to discover and characterize planets around stars spanning a wide range of masses. Of the 1887 confirmed exoplanets listed in the NASA Exoplanet Archive $^{14}$, only $104(5.5 \%)$ are around stars with masses

\footnotetext{
* Based on observations obtained with the Hungarian-made Automated Telescope Network. Based in part on observations made with the Keck-I telescope at Mauna Kea Observatory, HI (Keck time awarded through NASA programs $\mathrm{N} 029 \mathrm{Hr}, \mathrm{N} 108 \mathrm{Hr}, \mathrm{N} 154 \mathrm{Hr}$ and $\mathrm{N} 130 \mathrm{Hr}$ and $\mathrm{NOAO}$ programs A289Hr, and A284Hr). Based in part on observations made with the Nordic Optical Telescope, operated on the island of La Palma jointly by Denmark, Finland, Iceland, Norway, and Sweden, in the Spanish Observatorio del Roque de los Muchachos of the Instituto de Astrofísica de Canarias. Based in part on observations obtained with the Tillinghast Reflector $1.5 \mathrm{~m}$ telescope and the $1.2 \mathrm{~m}$ telescope, both operated by the Smithsonian Astrophysical Observatory at the Fred Lawrence Whipple Observatory in Arizona.

12 Alfred P. Sloan Research Fellow.

13 Packard Fellow.

${ }^{14}$ http://exoplanetarchive.ipac.caltech.edu, accessed 2015 August 27.
}

greater than $1.4 M_{\odot}$. The majority of these are evolved subgiant and giant stars whose planets were discovered through radial velocity (RV) surveys (the so-called "Retired A Star" surveys; e.g., Johnson et al. 2011; Wittenmyer et al. 2011; Sato et al. 2012), or moderately evolved stars with planets discovered by photometric transit surveys (e.g., HAT-P-49, Bieryla et al. 2014, and KELT-7, Bieryla et al. 2015, among others).

Finding planets around massive stars via RVs is challenging. The high surface temperatures of main sequence stars with $M \gtrsim 1.4 M_{\odot}$ leads to a high ionization fraction for most elements in their atmospheres and, as a result, their optical spectra have relatively few deep absorption lines that can be used for precise RV measurements. Moreover, unlike lower mass stars which lose angular momentum via magnetized stellar winds, and thus have surface rotation rates that decrease with increasing age, higher mass stars do not lose a significant amount of angular momentum over their main sequence lifetimes, and thus generally rotate at rapid 
velocities, often exceeding $100 \mathrm{~km} \mathrm{~s}^{-1}$ (e.g., Royer et al. 2007). The rapid rotation broadens the absorption lines in the stellar spectra, further reducing the precision with which their RVs may be measured. For typical A stars, the combined effects limit the per-point RV precision to a few $100 \mathrm{~m} \mathrm{~s}^{-1}$ at best. In order to overcome this limitation, several RV surveys have targeted evolved stars, thought to have been A stars when on the main sequence, which have lower surface temperatures and slower rotation rates compared to their main sequence counterparts.

Results from the Retired A Star RV surveys indicate that these stars have a population of planets that is significantly different from the planets around main sequence $F, G$ and $\mathrm{K}$ stars (e.g., Bowler et al. 2010). In particular, the stars targeted by these surveys appear to host giant planets significantly more often than $\mathrm{F}, \mathrm{G}$, and $\mathrm{K}$ stars, with a markedly different period distribution as well. This conclusion has not been without controversy, however, with both the masses of the host stars and the planetary nature of the detected periodic RV signals being called into question (Lloyd 2011, 2013, and Schlaufman \& Winn 2013; see, however, Johnson et al. 2013, 2014a who provide additional evidence for the retired-A-star nature of the targets). In any event, one might expect both stellar evolution (leading to the engulfment and/or evaporation of close-in planets), and the dynamical evolution of planetary systems through gravitational interactions, to result in systematic differences in the architectures of planetary systems around main sequence and post-main-sequence stars.

While there has been some success in finding planets around post-main-sequence stars with $M>1.4 M_{\odot}$ (bearing in mind the aforementioned caveats), finding planets around massive main sequence stars remains challenging. To date there are only 14 planets and low mass brown dwarfs reported around A- or B-type main sequence stars. Ten of these were discovered by direct imaging (including four around the A5V star HR 8799, Marois et al. 2008, one around the A6V star $\beta$ Pic, Lagrange et al. 2009, two around the A9V star HIP 73990, Hinkley et al. 2015, a candidate around the A7V star HD 169142, Biller et al. 2014, one around the B9V star HIP 78530, Lafrenière et al. 2011, and one around the B9IV star $\kappa$ And, Carson et al. 2013). These objects are on wide separations from their host stars, and are very massive (in most cases with $\mathrm{M}>10 M_{\mathrm{J}}$ ) and thus represent a substantially different population of planets from the closer-in and generally lower mass planets discovered through RVs or the transit technique. Moreover, the masses and radii of the directly imaged planets are not directly measured, but depend on theoretical planet evolution and atmosphere models which have large uncertainties due to the unknown initial conditions set by the planet formation process (e.g., Spiegel \& Burrows 2012), and due to the typically poorly constrained ages of the host stars (e.g., Baines et al. 2012).

The other four planets known around A stars were discovered through the transit technique (WASP-33, Collier Cameron et al. 2010b; Kepler-13A, Rowe et al. 2011; Shporer et al. 2011; Mazeh et al. 2012; and KOI-89 with two planets, Rowe et al. 2014). Finding planets around massive stars via transits is also more challenging than around less massive stars. Planets of a given size produce shallower transits around larger stars (though the transit durations are longer around more massive stars for a given orbital period, which compensates somewhat for the lower transit depths) making them harder to detect. And, once detected, it may not be possible to confirm the planets through the RV detection of the orbital wobble of their host stars for the reasons already discussed. None of the previously known transiting exoplanets (TEPs) around A stars were initially confirmed through the RV detection of the orbital wobbles of their host stars. Instead, Collier Cameron et al. (2010b) confirmed WASP-33b via Doppler tomography, Kepler-13Ab was confirmed through the photometric detection of Doppler beaming and the planet-induced tidal distortion of its host star, while KOI$89 \mathrm{~b}$ and KOI-89c were statistically validated by leveraging the low probability of false positives for objects with multiple periodic transit signals. RV-based measurements of the mass of WASP-33b have subsequently been reported by Kovács et al. (2013) and Lehmann et al. (2015), the latter finding a mass of $2.1 \pm 0.2 M_{\mathrm{J}}$ based on 248 spectroscopic observations.

While the rapid rotation of main sequence A stars hinders our ability to confirm and measure the masses of their TEPs through RV observations, it also presents a unique observational opportunity to characterize certain properties of these planetary systems. For very rapidly rotating stars the distortions in the spectral line profiles produced during planetary transits may be fully resolved without requiring very high resolution spectrographs, or even especially stable spectrographs (the motion of the planet shadow is measured in $\mathrm{km} \mathrm{s}^{-1}$ rather than $\mathrm{m} \mathrm{s}^{-1}$ ). This allows for a direct measurement of the track of the planet across the surface of the star with respect to the projected stellar spin axis. While the projected angle between the orbit of a TEP and the spin axis of its host star $\lambda$ may also be measured for slower rotating stars by detecting the anomalous Doppler shift that results from the line profile distortions during transit (the Rossiter-McLaughlin effect, e.g., Queloz et al. 2001), fully resolving the line profile distortions leads to a measurement of this angle that is both significantly more precise and more accurate.

Rapidly rotating stars are also more oblate than slower rotators, leading to rapid nodal precession for close-in planets on misaligned orbits. Johnson et al. (2015) leveraged the precision afforded by Doppler tomography to measure the change in $\lambda$ and the impact parameter $b$ of WASP-33b between 2008 and 2014, providing a direct measurement of the nodal precession rate of the system, and an observational constraint on the $J_{2}$ gravitational quadrupole moment of the star. Nodal precession has also been detected for Kepler-13Ab by Szabó et al. (2012) and by Masuda (2015) who measured a change in transit duration for this system via Kepler photometry.

The rotation-induced oblateness also leads to a non-uniform surface brightness profile of the star via the gravity-darkening effect. For planets on misaligned orbits this produces an asymmetric transit shape which may be used to measure the true (not projected) angle $\psi$ between the spin axis of the star and the orbital axis of the planet. This has been done using Kepler photometry by Masuda (2015) for Kepler- $13 \mathrm{Ab}$ and HAT-P-7b, and by Ahlers et al. (2015) for KOI-89b and KOI-89c.

In this paper we report the discovery of HAT-P-57b, a transiting giant planet around a rapidly rotating A8V star. With a rotation rate of $v \sin i=102.1 \pm 1.3 \mathrm{~km} \mathrm{~s}^{-1}$, HAT-P-57 is 
Table 1

Differential Photometry of HAT-P-57

\begin{tabular}{|c|c|c|c|c|c|}
\hline $\begin{array}{l}\mathrm{BJD}^{\mathrm{a}} \\
\quad(2,400,000+)\end{array}$ & $\mathrm{Mag}^{\mathrm{b}}$ & $\sigma_{\text {Phot }}$ & $\operatorname{Mag}\left(\right.$ orig) ${ }^{c}$ & Filter & Instrument \\
\hline 54998.84656 & 0.00470 & 0.00247 & $\cdots$ & $r$ & HATNet \\
\hline 55072.80545 & -0.00518 & 0.00237 & $\cdots$ & $r$ & HATNet \\
\hline 55067.87492 & -0.00243 & 0.00211 & $\cdots$ & $r$ & HATNet \\
\hline 55021.03497 & -0.00126 & 0.00251 & $\cdots$ & $r$ & HATNet \\
\hline 55077.73838 & -0.00658 & 0.00252 & $\cdots$ & $r$ & HATNet \\
\hline 55067.87893 & -0.00097 & 0.00212 & $\cdots$ & $r$ & HATNet \\
\hline 55025.96892 & 0.00174 & 0.00215 & $\ldots$ & $r$ & HATNet \\
\hline
\end{tabular}

Notes.

${ }^{\text {a }}$ Barycentric Julian Date calculated directly from UTC, without correction for leap seconds.

${ }^{b}$ The out-of-transit level has been subtracted. These values have been corrected for trends simultaneously with the transit fit for the follow-up data. For HATNet trends were filtered before fitting for the transit.

${ }^{\mathrm{c}}$ Raw magnitude values after correction using comparison stars, but without additional trend-filtering. We only report this value for the KeplerCam observations.

(This table is available in its entirety in machine-readable and Virtual Observatory (VO) forms.)

the most rapidly rotating star with a confirmed TEP, and it is also only the fourth A star with a confirmed TEP. In Section 2 we describe the observations leading to the discovery and characterization of this planetary system, the data are analyzed in Section 3, and we discuss the results in Section 4

\section{OBSERVATIONS}

\subsection{Photometry}

All time-series photometric data that we collected for HATP-57 are provided in Table 1. We discuss these observations below.

\subsubsection{Photometric Detection}

The star HAT-P-57 was observed by the HATNet wide-field photometric instruments (Bakos et al. 2004) between the nights of UT 2009 May 12 and UT 2009 September 14. A total of 622 observations of a $10^{\circ} 6 \times 10^{\circ} .6$ field centered at R.A. $=06^{\mathrm{h}} 24^{\mathrm{m}}$, decl. $=+30^{\circ}$ were made with the HAT-5 telescope in Arizona, and 3202 observations of this same field were made with the HAT-9 telescope in Hawaii (the count is after filtering 12 outlier measurements). We used a Sloan $r$ filter and an exposure time of $300 \mathrm{~s}$. Following Bakos et al. (2010) and Kovács et al. (2005), we reduced the images to trend-filtered light curves and searched these for periodic transit signals using the Box-fitting Least Squares algorithm (BLS; Kovács et al. 2002).

Transits were detected in the light curve of HAT-P-57 with a period of $P=2.4652950 \pm 0.0000032$ day. Figure 1 shows the phase-folded light curve together with our best-fit model. This same target has also been included in a list of TEP candidates published by the Super-WASP survey (Lister et al. 2007), and with a similar ephemeris, but was shortly thereafter set aside as a probable binary system based on follow-up spectroscopic observations showing that the star has a very rapid rotation (Collier Cameron et al. 2007). The target has also been independently identified as a TEP
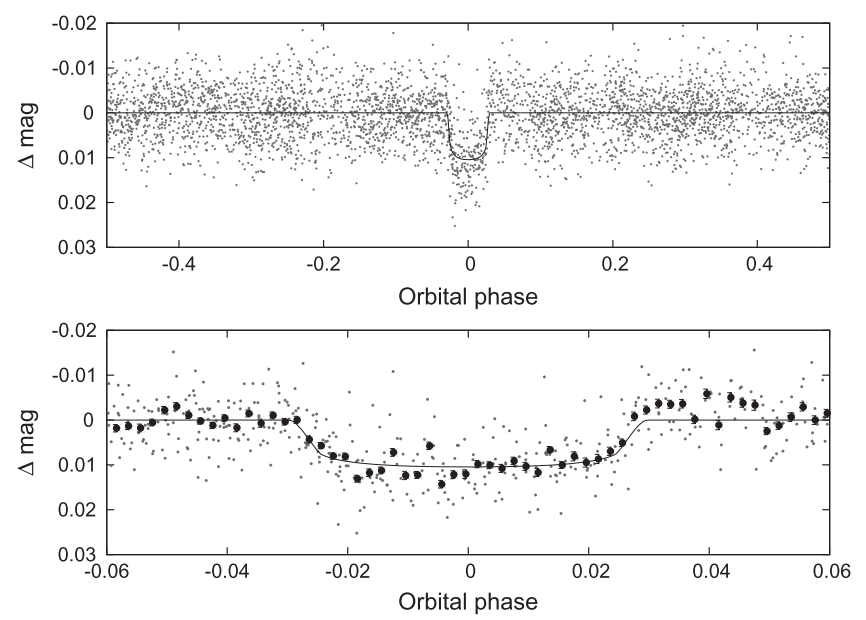

Figure 1. HATNet light curve of HAT-P-57 phase folded with the transit period. The top panel shows the unbinned light curve, while the bottom shows the region zoomed-in on the transit, with dark filled circles for the light curve binned in phase with a bin size of 0.002 . The solid line shows the model fit to the light curve.

candidate by the KELT survey (J. Pepper 2015, private communication; the KELT project is discussed in Siverd et al. 2012). We searched the light curve for additional transit signals or other periodic variations by running BLS and the Generalized Lomb-Scargle (Zechmeister \& Kürster 2009) algorithms on the residuals from the best-fit transit model. No additional transit signals were detected, however we do find two periodic signals at frequencies of $1.839 \mathrm{day}^{-1}$ (or its alias at $0.837 \mathrm{day}^{-1}$ ) and $2.030 \mathrm{day}^{-1}$ (or its alias at $1.026 \mathrm{day}^{-1}$ ) with false alarm probabilities of $10^{-6.1}$ and $10^{-2.1}$, respectively, and with peak-to-peak amplitudes of $1.7 \mathrm{mmag}$ and $1.3 \mathrm{mmag}$, respectively. The second frequency is identified after fitting and subtracting a sinusoid with a frequency of 1.839 day $^{-1}$. A further whitening cycle, with the 2.030 day $^{-1}$ signal also removed, reveals no other significant periods in the data (the highest signal in the final periodogram has a peak-to-peak 


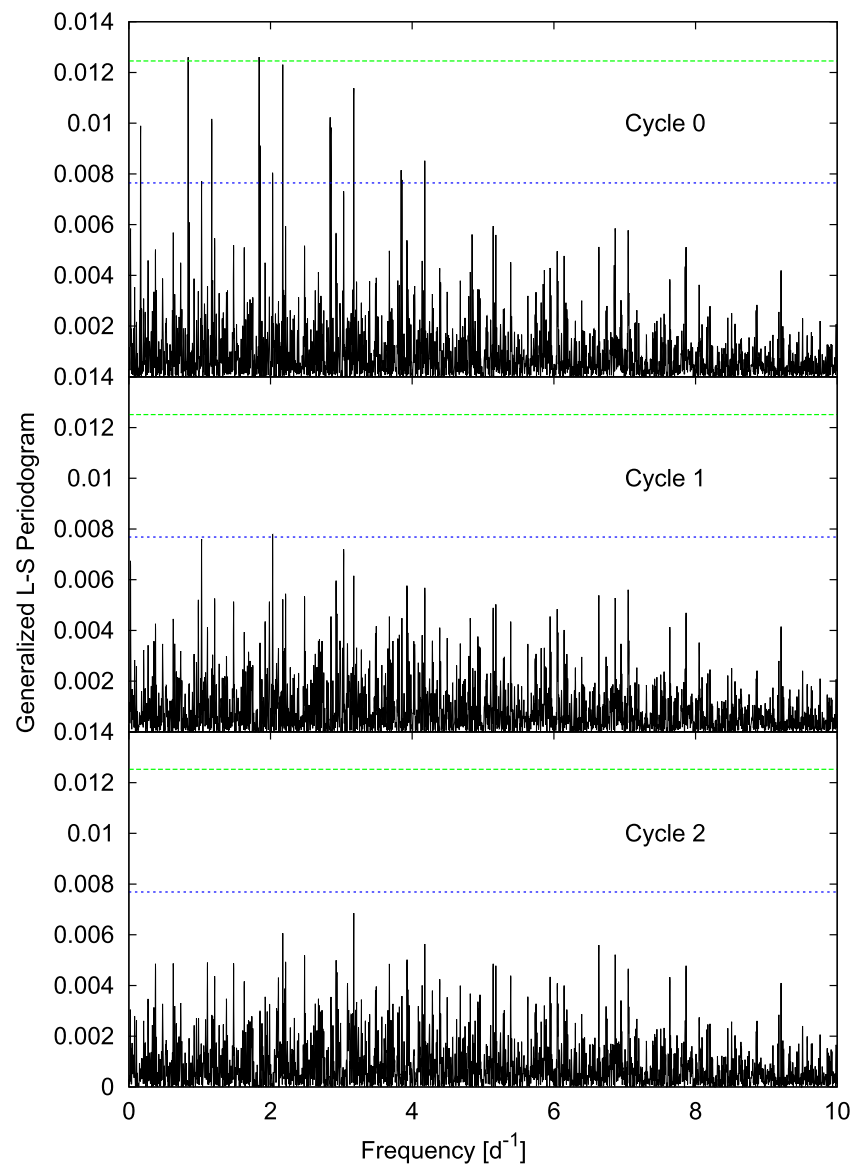

Figure 2. Generalized Lomb-Scargle periodogram of the HATNet light curve of HAT-P-57. The top panel is the periodogram of the original light curve with the transit signal removed, the middle panel is the periodogram after whitening the light curve at the highest power frequency identified the top periodogram $\left(1.839 \mathrm{day}^{-1}\right.$ or its alias at $\left.0.837 \mathrm{day}^{-1}\right)$, and the bottom panel is the periodogram after whitening the light curve at the highest power frequencies identified in the top and middle periodograms $\left(2.030\right.$ day $^{-1}$ or its alias at 1.026 day $\left.^{-1}\right)$. In each case the vertical axis is the unnormalized Generalized Lomb-Scargle periodogram given by Equation (5) of Zechmeister \& Kürster (2009). The dotted lines in each panel show the periodogram values corresponding to formal false alarm probabilities of $10^{-6}$ (upper lined) and $10^{-2}$ (lower lined). The periodograms are calculated up to a maximum frequency of $100 \mathrm{day}^{-1}$, but we only display them to a maximum frequency of 10 day $^{-1}$ because all significant power in the light curve is found below this frequency.

amplitude of $1.2 \mathrm{mmag}$ ). In each case the aliases are of comparable significance to the highest peak in the periodogram, and so we cannot determine whether the true primary frequency is $1.839 \mathrm{day}^{-1}$ or $0.837 \mathrm{day}^{-1}$, or whether the true secondary frequency is $2.030 \mathrm{day}^{-1}$ or $1.026 \mathrm{day}^{-1}$. Figure 2 shows the periodogram. One or both of these signals may be associated with the rotation period of the star, or they could correspond to gravity modes (all frequencies are too low for p-modes), in which case, given its surface temperature, HAT-P-57 would be a $\gamma$ Dor-type variable (cf., WASP-33 which shows both $\delta$-Scuti and $\gamma$-Dor variations). The temperature and surface gravity inferred for HAT-P-57 in Section 3.1 place it within both the classical $\delta$-Scuti and $\gamma$-Dor instability strips (e.g., Rodríguez \& Breger 2001, and Handler \& Shobbrook 2002, respectively), so pulsational variability is expected for HAT-P-57.

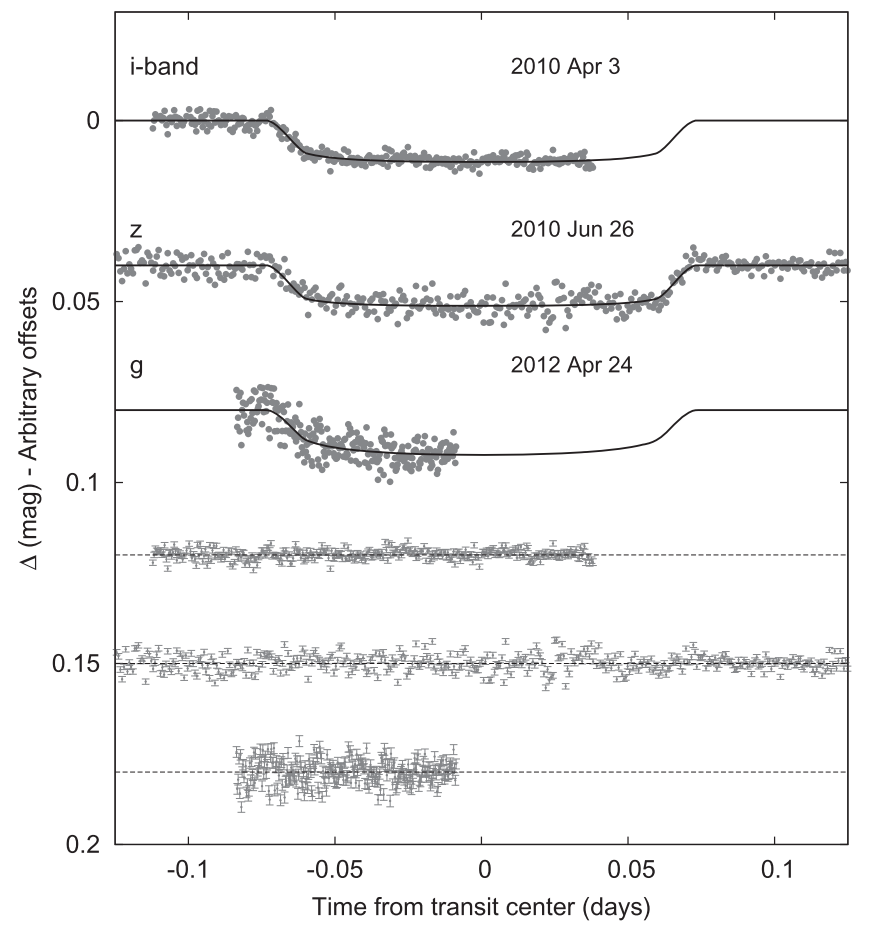

Figure 3. Unbinned transit light curves for HAT-P-57, acquired with KeplerCam at the FLWO $1.2 \mathrm{~m}$ telescope. The dates and band-passes are indicated. The light curve has been corrected for trends fit simultaneously with the transit model. Our best fit from the global modeling described in Section 3 is shown by the solid line. Residuals from the fit are displayed below the original light curves in the same order. The error bars represent the photon and background shot noise, plus the readout noise.

\subsubsection{Photometric Follow-up}

Photometric follow-up observations of HAT-P-57 were carried out with KeplerCam on the Fred Lawrence Whipple Observatory (FLWO) $1.2 \mathrm{~m}$ telescope. We observed ingress events on the nights of 2010 April 3 and 2012 April 24, in $i$ and $g$-bands respectively, and a full transit on the night of 2010 June 26 in $z$-band. The images were reduced to light curves following Bakos et al. (2010), including external parameter decorrelation performed simultaneously with the transit fit to remove systematic trends; the systematics-corrected light curves are shown in Figure 3. The rms of the residuals from our best-fit model varies from 1.4 to $3.4 \mathrm{mmag}$ for these data.

Additional photometric follow-up observations were carried out with the FLWO $1.2 \mathrm{~m}$ on the night of 2015 May 12 covering most of a predicted secondary eclipse event. The observations were performed in $z$-band and were used to constrain blend scenarios (Section 3.4).

\subsubsection{Adaptive Optics (AO) Imaging}

We obtained high-resolution imaging of HAT-P-57 on the night of UT 2011 June 22 using the Clio 2 near-IR imager (Freed et al. 2004) on the MMT $6.5 \mathrm{~m}$ telescope on Mt. Hopkins, in AZ. Observations in $H$-band and $L^{\prime}$-band were made using the AO system. Figure 4 shows the resulting images which reveal the presence of a binary pair of stars located 2". $667 \pm 0.001$ from HAT-P-57. The pair of stars is resolved into a $0^{\prime \prime} 225 \pm 0.002$ binary in the $L^{\prime}$ image. In $H$ band the two objects are not cleanly resolved, but the pointspread function (PSF) is clearly elongated. Applying aperture photometry to the $L^{\prime}$ observations we find that the two 

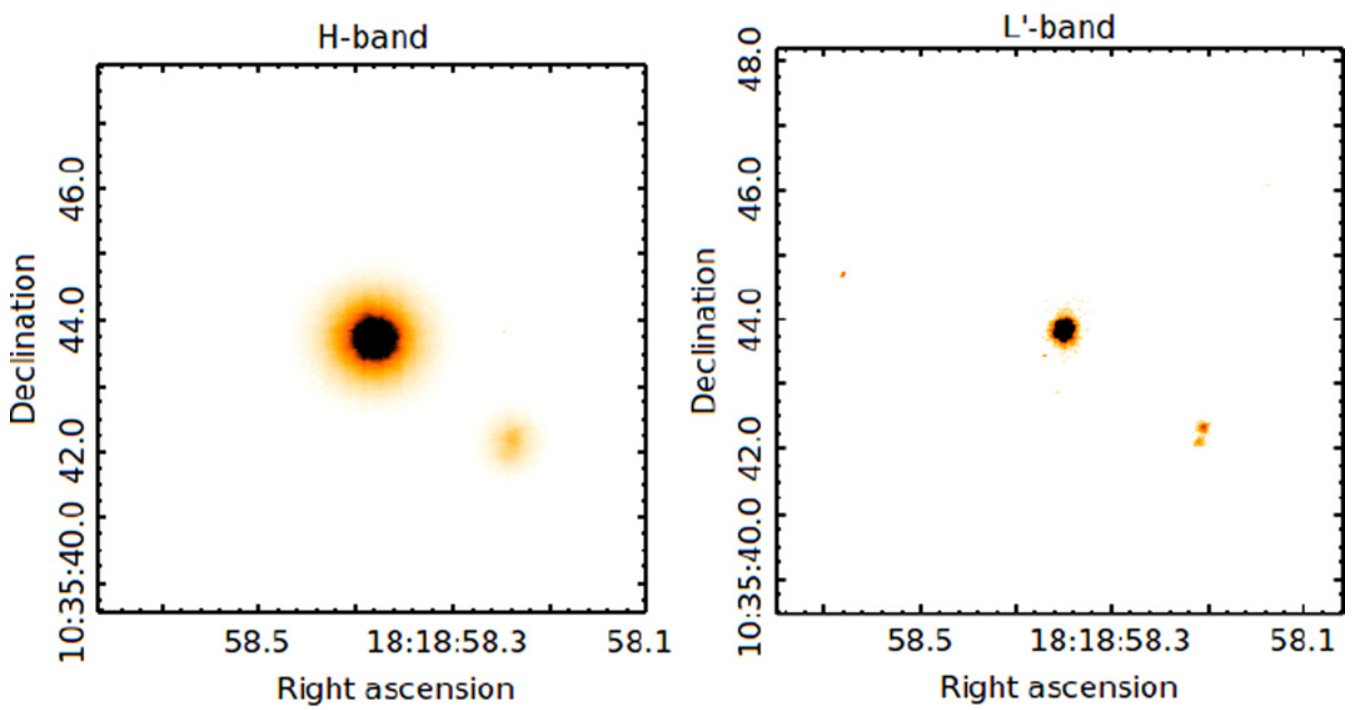

Figure 4. Representative $H$-band (left) and $L^{\prime}$-band (right) MMT/Clio2 images of HAT-P-57. A faint neighboring source is seen 2 ! $^{\prime} 7$ to the southwest of HAT-P-57. The $L^{\prime}$ image shows this source to itself be a binary object with two components separated by $0 . \prime 22$. Other brightness peaks in the $L^{\prime}$ image shown here are either hot pixels or cosmic ray hits.

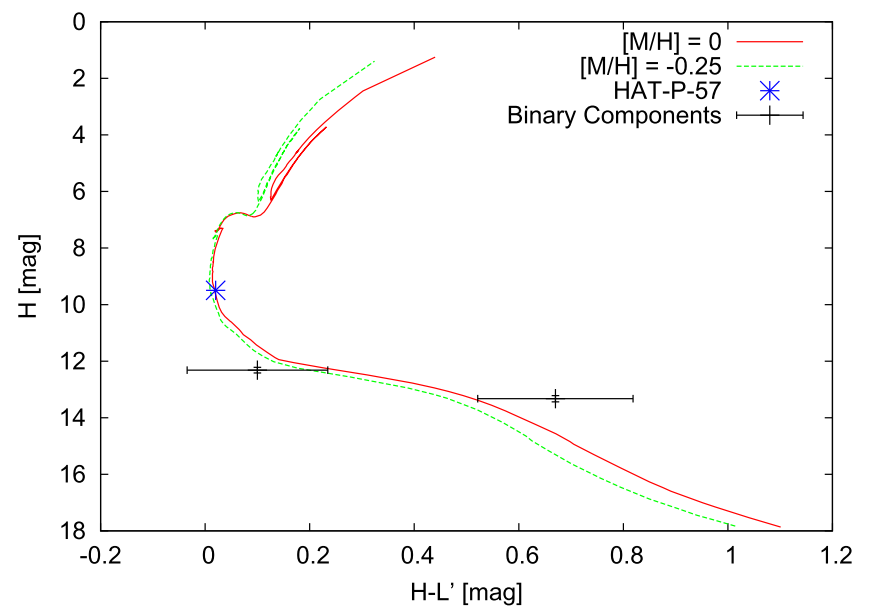

Figure 5. $H-L^{\prime}$ vs. $H$ color-magnitude diagram comparing the measured apparent magnitudes for HAT-P-57 and the components of the nearby binary system seen in Figure 4 to the 1 Gyr PARSEC isochrones (Bressan et al. 2012) shifted to the distance of HAT-P-57. The $L^{\prime}$ magnitude for HAT-P-57 is assumed based on its spectral type and measured $H$ magnitude. The components of the binary have photometry consistent with the isochrone, placing them at the same distance as HAT-P-57, so we conclude that they are likely to be physically associated with HAT-P-57.

components have $\Delta L^{\prime}$ magnitudes relative to HAT-P-57 of $\Delta L_{B}^{\prime}=2.72 \pm 0.09 \mathrm{mag}$ and $\Delta L_{C}^{\prime}=3.16 \pm 0.10 \mathrm{mag}$, respectively. To determine the relative $H$-band magnitudes we perform PSF fitting fixing the relative positions of the binary components to those from the $L^{\prime}$ images and using HAT-P-57 to define the PSF. We find $\Delta H_{B}=2.82 \pm 0.10$ mag and $\Delta H_{C}=3.83 \pm 0.11 \mathrm{mag}$.

Figure 5 shows the location of the two binary components, together with HAT-P-57, on a $H-L^{\prime}$ versus $H$ CMD. We also show 1.0 Gyr isochrones from the PARSEC model (Bressan et al. 2012) with metallicities of [M/ $H]=-0.25$ and $[M / H]=0.0$, and shifted to the distance of HAT-P-57 inferred in Section 3.1. We show the PARSEC model isochrones, rather than the $\mathrm{Y}^{2}$ isochrones which are used in Section 3.1 to determine the physical parameters of
HAT-P-57, because the PARSEC models provide a better match to the NIR photometry of M dwarf stars. The three stars are consistent with being on the same isochrone, so we conclude that the binary objects are likely to be physically associated with HAT-P-57. Assuming this is the case, we adopt the names HAT-P-57B and HAT-P-57C for the components of the binary objects, and estimate their masses to be $0.61 \pm 0.10 M_{\odot}$ and $0.53 \pm 0.08 M_{\odot}$, respectively. The 0 ". $225 \pm 0.002$ angular separation between HAT-P-57B and HAT-P-57C corresponds to a projected physical separation of $68 \pm 3 \mathrm{AU}$, and approximate orbital period of 500 years (assuming the projected separation corresponds to the physical semimajor axis of the orbit), while the 2 ". $667 \pm 0.001$ separation between the binary objects and HAT-P-57 corresponds to a projected physical separation of $800 \pm 30 \mathrm{AU}$ and approximate orbital period of 14,000 year.

Note that although we do not spatially resolve the binary object from HAT-P-57 in any of our photometric light curves, the Doppler tomography observations prove that the transiting component is orbiting the bright A star rather than either of the fainter components (Section 3.3). We also note that even without the Doppler tomography observations we would still be able to draw this conclusion as the binary object is too faint (in the optical), and its components are too red, to be responsible for the $1 \%$ transits seen consistently with KeplerCam in the $g, i$ and $z$-bands. Given the $H-L^{\prime}$ colors of the binary objects, we expect HAT-P-57B to be $6.2 \mathrm{mag}$ fainter than HAT-P-57 in $g$-band, $4.4 \mathrm{mag}$ fainter than HAT-P-57 in $i$-band, and $4.0 \mathrm{mag}$ fainter in $z$-band. Even if HAT-P-57B were totally eclipsed, the blended eclipse depth of $0.3 \%$ in $g$ would be too shallow to produce the observed $1 \%$ deep transits. Moreover, transits in $i$ and $z$ would be significantly deeper than in $g$, which is not what we observe.

Figure 6 shows the approximate $5 \sigma$ detection limits for any additional companions to HAT-P-57 in the $H$ and $L^{\prime}$-bands as a function of angular separation. These are estimated as five times the standard deviation of the pixel values in circular 


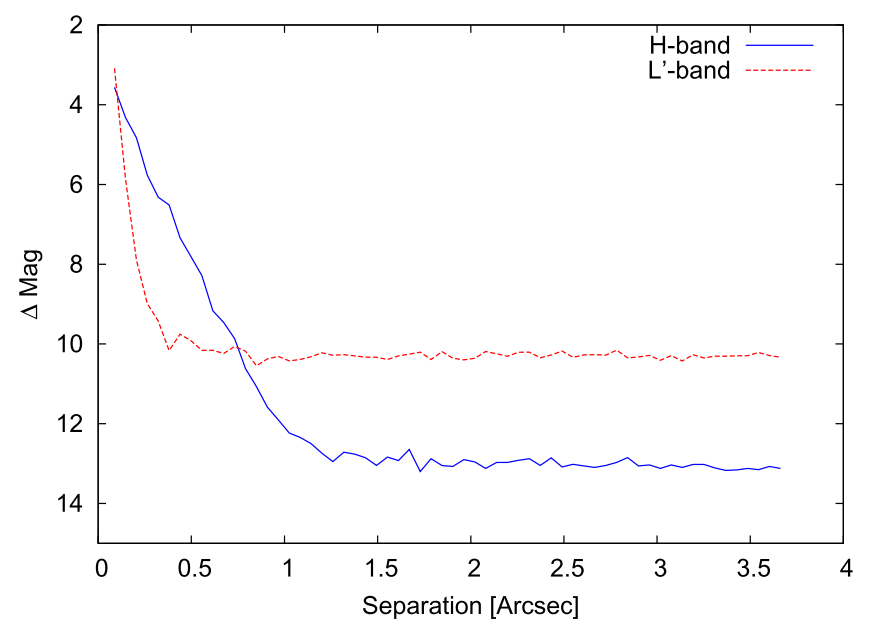

Figure 6. $5 \sigma$ detection limit for any additional companions to HAT-P-57 as a function of angular separation from HAT-P-57. These curves are based on the observations described in Section 2.1.3.

annuli centered on HAT-P-57, relative to the peak pixel value of HAT-P-57.

\subsection{Spectroscopy}

We carried out spectroscopic observations of HAT-P-57 between UT 2010 April 5 and UT 2010 July 1 with the Tillinghast Reflector Echelle Spectrograph (TRES; Füresz 2008) on the $1.5 \mathrm{~m}$ Tillinghast Reflector at FLWO. We also obtained spectra of HAT-P-57 on UT 2010 July 1-3 with the Fiber-fed Echelle Spectrograph (FIES; Telting et al. 2014) on the $2.56 \mathrm{~m}$ Nordic Optical Telescope (NOT) at the Observatorio del Roque de los Muchachos, on La Palma, Spain. Additional spectra were obtained using HIRES (Vogt et al. 1994) on the Keck-I $10 \mathrm{~m}$ telescope between UT 2010 June 27 and UT 2012 March 10. A total of 24 HIRES observations were collected during this time period, including 14 observations made through the $\mathrm{I}_{2}$ cell (e.g., Marcy \& Butler 1992), and 10 observations without the $\mathrm{I}_{2}$ cell. These latter observations were obtained on the night of UT 2010 June 27, primarily during a planetary transit (Section 3.3 discusses the analysis of these observations in more detail).

The TRES and FIES observations were reduced to initial $\mathrm{RVs}$, bisector spans (BSs) and stellar atmospheric parameters following Buchhave et al. (2010). Higher precision stellar atmospheric parameters were also measured from these observations using the Stellar Parameter Classification program (SPC; Buchhave et al. 2012). These measurements clearly indicate that HAT-P-57 is a rapidly rotating star with $v \sin i \gtrsim 100 \mathrm{~km} \mathrm{~s}^{-1}$, and a surface temperature hotter than $7000 \mathrm{~K}$. Due to the very broad absorption lines, however, the surface gravity cannot be reliably determined from the spectra with these techniques. Finally we note that the spectra do not appear to be composite, and also show no large RV variations. The five TRES RVs have an rms scatter of $2.2 \mathrm{~km} \mathrm{~s}^{-1}$, while the three FIES RVs have an rms scatter of $0.35 \mathrm{~km} \mathrm{~s}^{-1}$. The difference in precision is largely due to using a single spectral order to measure the TRES RVs, compared to five orders used for FIES (a multi-order analysis of the TRES data would yield more precise measurements, but given the lower $\mathrm{S} / \mathrm{N}$ of the

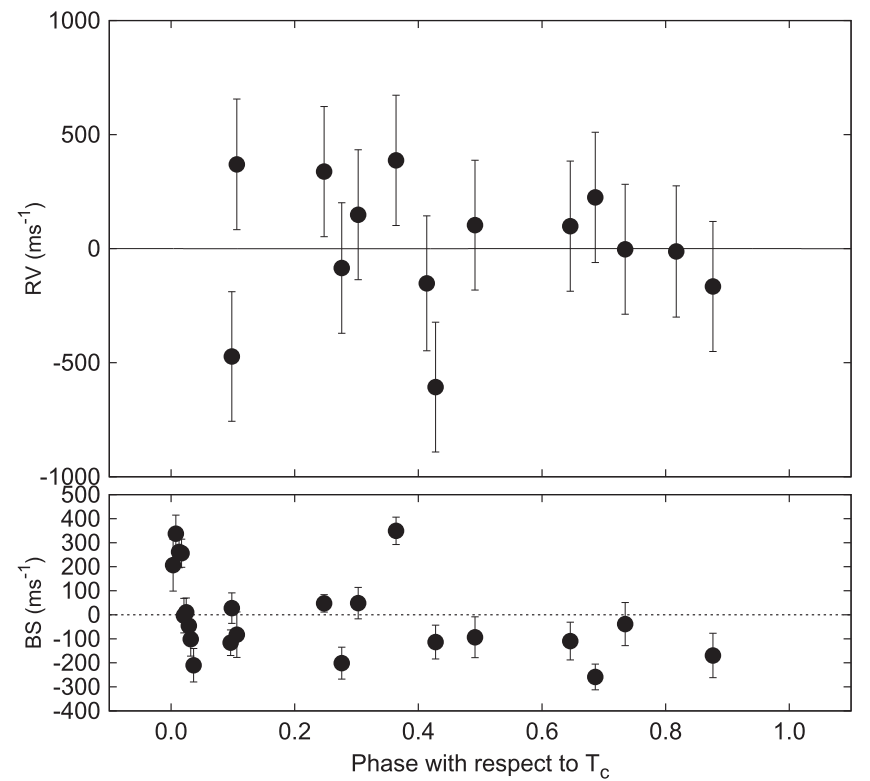

Figure 7. Top panel: RV measurements from Keck-I/HIRES computed using the $\mathrm{I}_{2}$-Doppler method, and shown as a function of orbital phase. Zero phase corresponds to the time of mid-transit. The center-of-mass velocity has been subtracted. The error bars include a "jitter" component $\left(312 \pm 70 \mathrm{~m} \mathrm{~s}^{-1}\right)$ added in quadrature to the formal errors. Due to the large scatter in the velocities resulting from the rapid rotation of the host star we do not detect the orbital variation of the star due to the planet. Based on these observations we place a $95 \%$ confidence upper limit on the orbital semi-amplitude of $K<215.2 \mathrm{~m} \mathrm{~s}^{-1}$. Bottom panel: bisector spans (BS). These are shown for the $\mathrm{I}_{2}$-free observations as well as the observations taken with the $\mathrm{I}_{2}$-cell in. A zoom-in on the in-transit measurements is shown in Figure 8. Note the different vertical scales of the panels.

spectra compared to those from FIES, we expect the scatter would still exceed that of the FIES RVs).

Wavelength calibrated spectra were extracted from the HIRES echelle images using the reduction pipeline of the California Planet Search team. The $14 \mathrm{I}_{2}$-in observations were reduced to relative RVs in the barycentric frame of the Solar System following the method of Butler et al. (1996). For this we made use of the highest $\mathrm{S} / \mathrm{N}$ out-of-transit $\mathrm{I}_{2}$-free observation as a template. These are shown phase-folded with the orbital ephemeris in Figure 7. We also measured spectral line BSs from the $\mathrm{I}_{2}$-free blue orders for 22 of the observations following Torres et al. (2007). Wavelength extracted spectra were not available for two observations and were excluded from the BS analysis. The BS values are also shown in Figure 7. Our procedure for measuring the BSs involves crosscorrelating the observed spectra against a synthetic template with atmospheric parameters similar to those measured for HAT-P-57. We used these same cross-correlations to measure the barycentric-corrected RVs for the spectra, including the 10 $\mathrm{I}_{2}$-free observations made on UT 2010 June 27 . Due to the slitfed nature of HIRES, and the lack of a simultaneously obtained wavelength calibration reference, the RV precision from this CCF method is substantially lower than the precision obtained from the standard $\mathrm{I}_{2}$ Doppler pipeline. More precise CCF-based RVs were also measured from the $10 \mathrm{I}_{2}$-free observations using both the blue and green spectral orders. Table 2 gives the relative RV measurements obtained with the $I_{2}$ Doppler pipeline, the RV measurements obtained from the CCFs, and the BSs for the HIRES observations. 
Table 2

Radial Velocities and Bisector Span Measurements of HAT-P-57 from Keck-I/HIRES

\begin{tabular}{|c|c|c|c|c|c|c|c|c|c|}
\hline $\begin{array}{l}\text { BJD }^{a} \\
(2,455,000+)\end{array}$ & $\begin{array}{l}\mathrm{RV} \mathrm{I}_{2}{ }^{\mathrm{b}} \\
\left(\mathrm{m} \mathrm{s}^{-1}\right)\end{array}$ & $\begin{array}{l}\sigma_{\mathrm{RV} \mathrm{I}_{2}}{ }^{\mathrm{c}} \\
\left(\mathrm{m} \mathrm{s}^{-1}\right)\end{array}$ & $\begin{array}{l}\mathrm{RV} \mathrm{CCF} \mathrm{B}^{\mathrm{d}} \\
\left(\mathrm{km} \mathrm{s}^{-1}\right)\end{array}$ & $\begin{array}{r}\sigma_{\mathrm{RV} \mathrm{CCF} \mathrm{B}} \\
\left(\mathrm{km} \mathrm{s}^{-1}\right)\end{array}$ & $\begin{array}{r}\mathrm{RV} \text { CCF B }+\mathrm{G}^{\mathrm{e}} \\
\left(\mathrm{km} \mathrm{s}^{-1}\right)\end{array}$ & $\begin{array}{r}\sigma_{\mathrm{RV} \mathrm{CCF} \mathrm{B}+\mathrm{G}} \\
\quad\left(\mathrm{km} \mathrm{s}^{-1}\right)\end{array}$ & $\begin{array}{r}\text { BS } \\
\left(\mathrm{m} \mathrm{s}^{-1}\right)\end{array}$ & $\begin{array}{r}\sigma_{\mathrm{BS}} \\
\left(\mathrm{m} \mathrm{s}^{-1}\right)\end{array}$ & Phase $^{\mathrm{f}}$ \\
\hline 374.81081 & $\cdots$ & $\cdots$ & -8.45 & 0.97 & -7.30 & 0.74 & 206 & 108 & 0.003 \\
\hline 374.82136 & $\cdots$ & $\cdots$ & -9.75 & 1.02 & -8.49 & 0.81 & 338 & 77 & 0.008 \\
\hline 374.83442 & $\cdots$ & $\ldots$ & -9.49 & 0.99 & -8.40 & 0.77 & 261 & 66 & 0.013 \\
\hline 374.84418 & $\cdots$ & $\cdots$ & -10.87 & 0.86 & -9.56 & 0.74 & 256 & 58 & 0.017 \\
\hline 374.85378 & $\ldots$ & $\ldots$ & -10.31 & 0.91 & -9.03 & 0.75 & -3 & 72 & 0.021 \\
\hline 374.86293 & $\cdots$ & $\ldots$ & -9.41 & 0.97 & -8.42 & 0.75 & 10 & 60 & 0.024 \\
\hline 374.87329 & $\cdots$ & $\cdots$ & -8.92 & 0.97 & -7.90 & 0.74 & -46 & 43 & 0.029 \\
\hline 374.88075 & $\cdots$ & $\cdots$ & -7.84 & 0.83 & -6.75 & 0.67 & -102 & 70 & 0.032 \\
\hline 374.89223 & $\ldots$ & $\ldots$ & -8.13 & 0.95 & -6.85 & 0.79 & -210 & 70 & 0.036 \\
\hline 375.04019 & $\cdots$ & $\cdots$ & -7.45 & 1.09 & -6.74 & 0.77 & -116 & 53 & 0.096 \\
\hline 375.04462 & -492 & 45 & -7.99 & 1.08 & $\ldots$ & $\ldots$ & 28 & 64 & 0.098 \\
\hline 375.85754 & -626 & 48 & -8.68 & 0.99 & $\ldots$ & $\cdots$ & -114 & 70 & 0.428 \\
\hline 467.82924 & -22 & 49 & -8.66 & 1.00 & $\cdots$ & $\cdots$ & -39 & 90 & 0.734 \\
\hline 607.15061 & 319 & 52 & -9.34 & 1.00 & $\cdots$ & $\cdots$ & 48 & 36 & 0.247 \\
\hline 608.13252 & 80 & 53 & -9.03 & 1.06 & $\cdots$ & $\cdots$ & -109 & 79 & 0.646 \\
\hline 612.15141 & -104 & 57 & -7.94 & 0.90 & $\cdots$ & $\cdots$ & -201 & 66 & 0.276 \\
\hline 613.16261 & 206 & 54 & -7.09 & 1.01 & $\cdots$ & $\cdots$ & -259 & 53 & 0.686 \\
\hline 699.91700 & -185 & 51 & -7.66 & 0.93 & $\ldots$ & $\ldots$ & -169 & 93 & 0.876 \\
\hline 701.11852 & 368 & 54 & -9.37 & 0.96 & $\cdots$ & $\cdots$ & 350 & 57 & 0.364 \\
\hline 703.89871 & 84 & 48 & -9.36 & 0.86 & $\cdots$ & $\cdots$ & -93 & 85 & 0.491 \\
\hline 705.89808 & 130 & 50 & -9.17 & 0.86 & $\cdots$ & $\cdots$ & 49 & 65 & 0.302 \\
\hline 707.87969 & 351 & 58 & -6.93 & 1.04 & $\cdots$ & $\cdots$ & -83 & 95 & 0.106 \\
\hline 879.73744 & -31 & 64 & $\ldots$ & $\ldots$ & $\cdots$ & $\cdots$ & $\ldots$ & $\cdots$ & 0.817 \\
\hline 997.07656 & -171 & 95 & $\cdots$ & $\cdots$ & $\cdots$ & $\cdots$ & $\cdots$ & $\cdots$ & 0.413 \\
\hline
\end{tabular}

Notes.

a Barycentric Julian Date calculated directly from UTC, without correction for leap seconds.

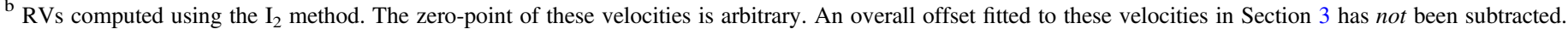
Spectra obtained without the $\mathrm{I}_{2}$-cell in do not have an RV measurement listed in this column.

${ }^{c}$ Internal errors excluding the component of astrophysical jitter considered in Section 3 .

${ }^{\mathrm{d}}$ RVs computed using the CCF method, applied only to the $\mathrm{I}_{2}$-free blue spectral orders. Note that the units here are $\mathrm{km} \mathrm{s}^{-1}$ rather than $\mathrm{m} \mathrm{s}^{-1}$.

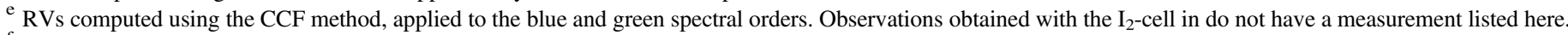

${ }^{\mathrm{f}}$ Orbital phase, with phase zero corresponding to mid-transit.

The CCF-based RVs and the BSs measured from the intransit HIRES observations both show evidence of the Rossiter-McLaughlin effect (Figure 8). Note that the sense of the variation seen in both indicators is consistent. For the BS values plotted, we are using the definition

$$
B S=R V_{\mathrm{CCF}, \min }-R V_{\mathrm{CCF}, \max }
$$

where $R V_{\mathrm{CCF}, \min }$ is the bisector velocity at the low CCF value (i.e., in the wings of the absorption line), while $R V_{\mathrm{CCF} \text {,max }}$ is the bisector velocity at the high CCF value (in the core of the line). A positive BS indicates that the core of the line is blueshifted compared to the wings of the line. Thus with this definition, we expect the BS and RV variations to be anti-correlated. Rather than attempting to measure the projected spin-orbit alignment angle $\lambda$ from these observations we perform a Doppler tomography analysis of the line profile distortions in Section 3.3. In Figure 8 we also show the approximate expected $\mathrm{RV}$ variation due to the RM effect calculated using the ARoME package (Boué et al. 2013) for the maximum posterior probability solution determined from the line profile modeling. This model underpredicts the anomalous Doppler shift, and if we attempt to fit the model directly to the observations then the results require a very high value for $v \sin i\left(175 \mathrm{~km} \mathrm{~s}^{-1}\right)$, which is completely inconsistent with the width of the line profiles seen in the HIRES spectra. The model, however, is not applicable to very high rotation rates $\left(v \sin i>20 \mathrm{~km} \mathrm{~s}^{-1}\right)$, and so such a discrepancy is not unexpected.

\section{ANALYSIS}

\subsection{Stellar Parameters}

We measured the stellar atmospheric parameters for HAT-P57 in two ways. First we analyzed both the HIRES $I_{2}$-free observations and the FIES observations with SPC. For the second method we performed a $\chi^{2}$ comparison of synthetic templates from the Pollux database (Palacios et al. 2010) to the HIRES observations.

The two HIRES orders covered by the SPC library yield substantially different results for the temperature, metallicity and surface gravity. For one of the orders we find $T_{\text {eff }}=6620 \mathrm{~K}, \log g=3.39,[M / H]=-1.08$ and $v \sin i=$ $101.7 \mathrm{~km} \mathrm{~s}^{-1}$. The normalized CCF has a peak height of 0.978 indicating a good match between the observations and the synthetic template. For the other order we find $T_{\text {eff }}=8450 \mathrm{~K}, \log g=4.37,[M / H]=0.01$ and $v \sin i=$ $102.3 \mathrm{~km} \mathrm{~s}^{-1}$. The CCF in this case has a peak height of 0.984 , again indicating a good match. When the surface gravity is fixed to 4.20 , based on the $\mathrm{Y}^{2}$ stellar evolution models, and using the transit-based stellar density and the effective 


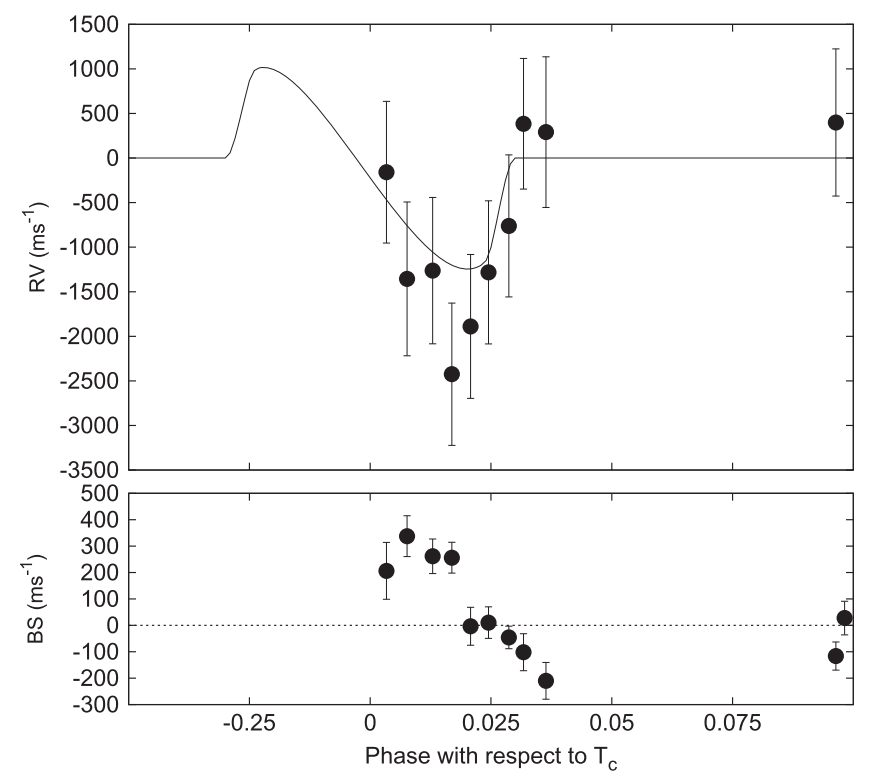

Figure 8. Top panel: CCF-based RV measurements from the $I_{2}$-free Keck-I/ HIRES observations made during (first seven observations) and just after transit (last three observations), shown as a function of orbital phase. The Rossiter-McLaughlin effect with an amplitude of $\sim 2 \mathrm{~km} \mathrm{~s}^{-1}$ is seen during transit. For reference we also show the predicted RV variation from the ARoME model (Boué et al. 2013) for the maximum posterior probability solution to the spectral line profiles (Figure 9). The model underpredicts the measured variation, but since the star is rotating much faster than the maximum velocity at which the model is applicable, such a discrepancy may be expected. Bottom panel: the BSs (computed for $\mathrm{I}_{2}$-in observations as well as the $\mathrm{I}_{2}$-free observations) also show an anomalous variation during transit due to the spectral line profile distortion caused by the TEP.

temperature and metallicities estimated from the spectra (Section 3.2; note that we find that the surface gravity from this analysis is quite well constrained despite the very large initial uncertainty in the temperature and metallicity), we find temperatures of $T_{\text {eff }}=7250 \mathrm{~K}$ and $T_{\text {eff }}=8280 \mathrm{~K}$, and metallicities of $[M / H]=-0.70$ and $=-0.03$ from the respective orders. Due to the lack of consistency between the two orders, we conclude that the spectral overlap between HIRES and the SPC library is too small, in this case, for a reliable determination of the atmospheric parameters.

When we analyzed the three FIES observations with SPC we find $T_{\text {eff }}=6830 \pm 120 \mathrm{~K}, \log g=2.95 \pm 0.15, \quad[M / H]=$ $-0.82 \pm 0.04$, and $v \sin i=103.7 \pm 0.9 \mathrm{~km} \mathrm{~s}^{-1}$, with a cross-correlation peak-height of 0.914 . The uncertainties are the standard deviation of the measurements from the three separate observations. When the surface gravity is fixed to $\log g=4.20$, we find $T_{\text {eff }}=7440 \pm 80 \mathrm{~K}, \quad[M / H]=$ $-0.39 \pm 0.05, \quad v \sin i=102.8 \pm 1.1 \mathrm{~km} \mathrm{~s}^{-1}$, and a crosscorrelation peak-height of 0.910 . The listed uncertainties reflect the precision, but not the accuracy, of the measurements. In particular they do not account for the degeneracies between the parameters, which are more significant at such high rotation velocities than they are for slower rotating stars where the true SPC errors have been well calibrated.

For an A star like HAT-P-57 the wavelength range of 5050-5360 A used by SPC does not contain very many good absorption lines for determining the atmospheric parameters, resulting in significant degeneracies between the parameters. We therefore carried out a separate analysis of the Keck/ HIRES observations of HAT-P-57, focusing in this case on 18 blue orders covering the wavelength range 3840-4793 $\AA$. This range of the spectrum contains several Hydrogen Balmer lines, whose broad profiles constrain the temperature, as well as many ionized metal lines which are useful for determining both the metallicity and the temperature.

We first normalized the continua of the observed spectra by fitting polynomials in wavelength and order to the numerous continuum regions available for this rapidly rotating star. We then used the Pollux database (Palacios et al. 2010) to obtain a grid of synthetic high resolution spectra generated using the MARCS atmosphere models (Gustafsson et al. 2008). The grid spans the temperature range $6500-8000 \mathrm{~K}$ in $250 \mathrm{~K}$ steps, metallicities from $[M / H]=-1.0$ to 1.0 in 0.25 dex steps, and surface gravities of $\log g=4.0$ and $\log g=4.5$. We applied a rotational broadening kernel with $v \sin i=102.8 \mathrm{~km} \mathrm{~s}^{-1}$, based on the SPC analysis of the FIES data, and assuming a linear limb darkening law with a coefficient of 0.6 , to the templates and also applied the same continuum normalization procedure as performed on the observed spectra. Working order by order, we then cross-correlated each template against the observed spectrum to determine the redshift, applied the redshift to the template, interpolated the redshifted template to the wavelength grid of the observations, and measured the $\chi^{2}$ difference between the template and observations ignoring the edges of the order where the errors are high and the blazefunction and wavelength solution have systematic errors (the wavelength range to use was determined manually for each order). For each $\log g$ value we fit a polynomial to the $\chi^{2}-[M /$ $H]-T_{\text {eff }}$ surface to determine the $T_{\text {eff }}$ and $[M / H]$ values which minimize the total $\chi^{2}$ for a spectrum. For $\log g=4.0$ we find $T_{\text {eff }}=7476 \pm 11 \mathrm{~K}$ and $[M / H]=-0.2589 \pm 0.0037$, while for $\log g=4.5$ we find $T_{\text {eff }}=7541 \pm 10 \mathrm{~K}$ and $[M /$ $H]=-0.2337 \pm 0.0039$. The errors here are the standard deviation of the results, demonstrating that this procedure yields very consistent parameters from observation to observation. The real errors, however, are dominated by systematic errors in the models and the relatively low temperature and metallicity resolution of the grid. For simplicity we adopt the grid resolution for our estimated errors, with $T_{\text {eff }}=7500 \pm 250 \mathrm{~K}$, and $[M / H]=-0.25 \pm 0.25$. These results are consistent with the parameters estimated from the FIES spectra with SPC, and are the parameters that we adopt for the remainder of this paper.

We note that in appearance the spectra are consistent with a late A or early F classification. Based on the temperaturespectral type scale from Pecaut \& Mamajek (2013), a temperature of $T_{\text {eff }}=7500 \mathrm{~K}$ corresponds to a spectral type of A8.

The adopted values for $T_{\text {eff }}$ and $[M / H]$, together with the transit-based mean stellar density (Section 3.2), were then combined with the Yonsei-Yale $\left(\mathrm{Y}^{2}\right)$ stellar evolution models (Yi et al. 2001) to determine the mass, radius, luminosity and age of HAT-P-57. Figure 14 compares the measured values of $T_{\text {eff }}$ and $\rho_{\star}$ to the isochrones. Table 3 lists the observed and derived stellar parameters. We find that HAT-P-57 has a mass of $1.47 \pm 0.12 M_{\odot}$, a radius of $1.500 \pm 0.050 R_{\odot}$, and is at a reddening- and blend-corrected distance of $303 \pm 13$ pc.

\subsection{Modeling of RVs and Light Curves}

We modeled the trend-filtered HATNet light curve and the KeplerCam light curves of HAT-P-57, together with the Keck/ HIRES $\mathrm{I}_{2}$ RVs following the methods described by Bakos et al. 
Table 3

Stellar Parameters for HAT-P-57

\begin{tabular}{|c|c|c|}
\hline Parameter & Value & Source \\
\hline \multicolumn{3}{|c|}{ Identifying Information } \\
\hline R.A. (h:m:s) & $18^{\mathrm{h}} 18^{\mathrm{m}} 58^{\mathrm{s}} .32$ & 2MASS \\
\hline Decl. (d:m:s) & $+10^{\circ} 35^{\prime} 50^{\prime \prime} 3$ & 2MASS \\
\hline GSC ID & GSC 1014-00973 & GSC \\
\hline 2MASS ID & 2MASS $18185842+1035502$ & 2MASS \\
\hline SWASP ID & 1SWASP J181858.42 + 103550.1 & SWASF \\
\hline \multicolumn{3}{|c|}{ Spectroscopic properties } \\
\hline$T_{\text {eff } \star}(\mathrm{K})$ & $7500 \pm 250$ & HIRES + Pollux \\
\hline$[\mathrm{Fe} / \mathrm{H}]$ & $-0.25 \pm 0.25$ & HIRES+Pollux \\
\hline$v \sin i\left(\mathrm{~km} \mathrm{~s}^{-1}\right)$ & $102.1 \pm 1.3$ & HIRES \\
\hline$\gamma_{\mathrm{RV}}\left(\mathrm{km} \mathrm{s}^{-1}\right)$ & $-5.99 \pm 0.35$ & FIES \\
\hline \multicolumn{3}{|c|}{ Photometric properties } \\
\hline$B$ (mag) & $10.916 \pm 0.021$ & $\begin{array}{l}\text { APASS (via } \\
\text { URAT1) }\end{array}$ \\
\hline$V(\mathrm{mag})$ & $10.465 \pm 0.029$ & APASS \\
\hline$I$ (mag) & $10.007 \pm 0.063$ & TASS Mark IV \\
\hline$g(\mathrm{mag})$ & $10.741 \pm 0.074$ & APASS \\
\hline$r(\mathrm{mag})$ & $10.371 \pm 0.053$ & APASS \\
\hline$i$ (mag) & $10.282 \pm 0.034$ & APASS \\
\hline$J$ (mag) & $9.670 \pm 0.027$ & 2MASS \\
\hline$H$ (mag) & $9.497 \pm 0.029$ & 2MASS \\
\hline$K_{s}(\mathrm{mag})$ & $9.433 \pm 0.024$ & 2MASS \\
\hline \multicolumn{3}{|c|}{ Derived properties } \\
\hline$M_{\star}\left(M_{\odot}\right)$ & $1.47 \pm 0.12$ & Isochrones $+\rho_{x}$ \\
\hline$R_{\star}\left(R_{\odot}\right)$ & $1.500 \pm 0.050$ & $\begin{array}{r}+ \text { HIRES }+ \text { Pollux } \\
\text { Isochrones }+\rho_{\star}\end{array}$ \\
\hline$\rho_{\star}(\mathrm{cgs})$ & $0.615_{-0.036}^{+0.022}$ & $\begin{array}{c}+ \text { HIRES }+ \text { Pollux } \\
\text { Light Curves }\end{array}$ \\
\hline $\log g_{\star}(\mathrm{cgs})$ & $4.251 \pm 0.018$ & Isochrones $+\rho$ \\
\hline & & + HIRES + Pollux \\
\hline$L_{\star}\left(L_{\odot}\right)$ & $6.4 \pm 1.1$ & Isochrones $+\rho_{\star}$ \\
\hline & & + HIRES +Pollux \\
\hline$M_{V}(\mathrm{mag})$ & $2.70 \pm 0.19$ & Isochrones $+\rho$ \\
\hline$M_{K}(\mathrm{mag}, \mathrm{ESO})$ & $2.13 \pm 0.10$ & $\begin{array}{r}+ \text { HIRES }+ \text { Pollux } \\
\text { Isochrones }+\rho_{\star}\end{array}$ \\
\hline & & + HIRES + Pollux \\
\hline Age (Gyr) & $1.00_{-0.51}^{+0.67}$ & Isochrones $+\rho$ \\
\hline$A_{V}(\mathrm{mag})^{\mathrm{d}}$ & $0.38 \pm 0.12$ & $\begin{array}{l}+ \text { HIRES }+ \text { Pollux } \\
\text { Isochrones }+\rho_{\star}\end{array}$ \\
\hline Distance $(\mathrm{pc})^{\mathrm{e}}$ & $303 \pm 13$ & $\begin{array}{r}+ \text { HIRES }+ \text { Pollux } \\
\text { Isochrones }+\rho_{\star} \\
+ \text { HIRES }+ \text { Pollux }\end{array}$ \\
\hline
\end{tabular}

Notes.

${ }^{\mathrm{a}}$ HIRES + Pollux $=$ Based on a $\chi^{2}$ comparison between the extracted HIRES spectra and synthetics MARCS model atmosphere spectra (Gustafsson et al. 2008) from the Pollux database (Palacios et al. 2010) as discussed in Section 3.1.

${ }^{\mathrm{b}}$ Based on modeling the spectral line profiles as discussed in Section 3.3.

${ }^{\mathrm{c}}$ Isochrones $+\rho_{\star}+$ HIRES + Pollux $=$ Based on the $\mathrm{Y}^{2}$ isochrones (Yi et al. 2001), the stellar density used as a luminosity indicator, and the atmospheric parameter results.

${ }^{\mathrm{d}}$ Total $V$ band extinction to the star determined by comparing the catalog broadband photometry listed in the table to the expected magnitudes from the Isochrones $+\rho_{\star}+$ HIRES + Pollux model for the star, and accounting for blending from the known binary located 2!" 7 away from HAT-P-57. We use the Cardelli et al. (1989) extinction law.

${ }^{\mathrm{e}}$ Distance based on a comparison of the measured photometric magnitudes for HAT-P-57, corrected for blending from HAT-P-57A and HAT-P-57B and for reddening, to the predicted magnitudes from the stellar evolution models.
(2010) and Hartman et al. (2012). The light curves were fit using a Mandel \& Agol (2002) transit model with quadratic limb darkening coefficients fixed to the values adopted from Claret (2004). For the KeplerCam light curves we allowed for a quadratic trend in hour angle, and linear trends in three parameters describing the shape of the PSF. For the HATNet light curve we included a dilution factor to account for distortion of the transit signal due to the filtering procedure, and blending from neighboring stars in the low spatial resolution HATNet images. The RVs were included in the fit and modeled using a circular Keplerian orbit. The RV "jitter" term was also varied in the fit following Hartman et al. (2014). Note that there is no evidence that the low cadence $\mathrm{I}_{2} \mathrm{RV}$ observations are correlated in time, justifying the assumption of uncorrelated RV jitter. Although no orbital variation is detected, this procedure allows us to place an upper limit on the RV semiamplitude, and hence on the mass of HAT-P-57b.

To account for blending in the KeplerCam light curves from HAT-P-57B and HAT-P-57C we included contamination factors in each bandpass. We allowed these factors to vary in the fit with Gaussian priors which were estimated based on the measured $\Delta H$ and $\Delta L^{\prime}$ magnitudes, together with the PARSEC isochrones, and assuming the binary is physically associated with HAT-P-57. The adopted priors are listed in Table 4.

We used a Differential Evolution Markov Chain Monte Carlo (DEMCMC) simulation (ter Braak 2006) to explore the likelihood function and produce posterior distributions for all varied parameters. The parameters that we varied, together with their adopted priors, are listed in Table 4. The resulting Markov Chains were combined with the chains of stellar parameters produced in Section 3.1 to determine the radius, semimajor axis, and other physical and orbital parameters for HAT-P-57b. In particular we find that HAT-P-57b has a radius of $R_{P}=1.413 \pm 0.054 R_{\mathrm{J}}$, and we place a $95 \%$ confidence upper limit on its mass of $M_{P}<1.85 M_{\mathrm{J}}$. Table 5 lists these and other parameters for HAT-P-57b.

\subsection{Line Profile Modeling}

Due to the very rapid rotation and brightness of HAT-P-57, the in-transit Keck-I/HIRES observations are amenable to Doppler tomography (e.g., Collier Cameron et al. 2010b). Because of the rapid rotation, there are essentially no unblended lines in the spectrum of HAT-P-57. We therefore make use of the Least Squares Deconvolution method (Donati et al. 1997; see also Collier Cameron et al. 2010b who apply this method to observations of WASP-33) to extract the broadening profile from the blended spectrum. Rather than using a list of weighted delta-functions at known spectral features for the line pattern function, as done by Donati et al. (1997), we use the unbroadened MARCS-atmosphere synthetic template which provided the best match (after broadening) to the Keck-I/HIRES spectra (Section 3.1). The deconvolution is done on an order-by-order basis, and we then take the weighted average of 21 orders spanning $4000-5700 \AA$, excluding those containing deep Hydrogen Balmer lines or Ca II lines. The average broadening profiles are shown in Figure 9. The residuals from a quadratic limb-darkening rotational profile are shown in Figure 10. The TEP is clearly seen as the dip in Figure 9, or shadow in Figure 10, moving from $\Delta V \sim 15 \mathrm{~km} \mathrm{~s}^{-1}$ in the first observation near transit center to $\Delta V \sim 77 \mathrm{~km} \mathrm{~s}^{-1}$ shortly before egress. 
Table 4

MCMC State Variables and Priors

\begin{tabular}{|c|c|}
\hline Parameter & Prior \\
\hline \multicolumn{2}{|c|}{ Light curve $+\mathrm{RV}$ curve analysis } \\
\hline$T_{c, 0}(\text { days })^{\mathrm{a}}$ & uniform \\
\hline$T_{c, 888}(\text { days })^{\mathrm{a}}$ & uniform \\
\hline$\zeta / R_{\star}$ & uniform \\
\hline$R_{p} / R_{\star}$ & uniform \\
\hline$b^{2}$ & uniform with $0 \leqslant b^{2} \leqslant 1$ \\
\hline$K\left(\mathrm{~km} \mathrm{~s}^{-1}\right)$ & uniform with $K \geqslant 0$ \\
\hline$\gamma_{\text {rel }}$ HIRES & uniform \\
\hline RV jitter HIRES & uniform with $\sigma_{\mathrm{jitter}} \geqslant 0$ \\
\hline$f_{\text {blend,HN, } \mathrm{r}}{ }^{\mathrm{b}}$ & uniform with $0 \leqslant f_{\text {blend,HN }} \leqslant 1$ \\
\hline$f_{\text {blend,g }}{ }^{\mathrm{b}}$ & $N(0.9980,0.0026)^{\mathrm{c}}$ with $0 \leqslant f_{\text {blend,g }} \leqslant 1$ \\
\hline$f_{\text {blend,i }}{ }^{\mathrm{b}}$ & $N(0.9836,0.0088)^{\mathrm{c}}$ with $0 \leqslant f_{\text {blend, } \mathrm{i}} \leqslant 1$ \\
\hline$f_{\text {blend, },}{ }^{\mathrm{b}}$ & $N(0.973,0.011)^{\mathrm{c}}$ with $0 \leqslant f_{\text {blend, } \mathrm{z}} \leqslant 1$ \\
\hline$m_{0}{ }^{\mathrm{d}}$ & uniform, linearly optimized \\
\hline$c_{\mathrm{EPD}}{ }^{\mathrm{e}}$ & uniform, linearly optimized \\
\hline
\end{tabular}

Line profile analysis

$\begin{array}{ll}T_{c} \text { (days) } & N(2455113.48127,0.00048)^{\mathrm{c}} \\ P \text { (days) } & N(2.4652950,0.0000032)^{\mathrm{c}} \\ a / R_{\star} & N(5.825,0.090)^{\mathrm{c}} \\ R_{p} / R_{\star} & N(0.0968,0.0015)^{\mathrm{c}} \\ b^{2} & N(0.051,0.023)^{\mathrm{c}} \text { with } 0 \leqslant b^{2} \leqslant 1 \\ \lambda(\text { days }) & \text { uniform }-180 \leqslant \lambda<180 \\ v \sin i\left(\mathrm{~km} \mathrm{~s}^{-1}\right) & \text { uniform } \\ \Delta v_{0}\left(\mathrm{~km} \mathrm{~s}^{-1}\right)^{\mathrm{f}} & \text { uniform } \\ u_{1}^{\prime} & \text { uniform subject to } 0 \leqslant u_{1} \leqslant 1 \\ u_{2}^{\prime} & \text { uniform subject to } 0 \leqslant u_{2} \leqslant 1 \\ \rho^{\mathrm{g}} & \propto e^{-\rho / 200} \text { with } 0 \leqslant \rho \leqslant 200 \\ A^{\mathrm{g}} & \propto e^{-A / 0.0000002} \\ \alpha^{\mathrm{g}} & \propto 1 / \alpha \text { with } \alpha>0 \\ a_{\mathrm{LP}} & \text { uniform } \\ c_{\mathrm{LP}} & \text { uniform }\end{array}$

\section{Notes.}

${ }^{\mathrm{a}}$ The times of transit center for event number 0 (the first transit covered by our light curves) and event number 888 (the last transit covered by our light curves).

${ }^{\mathrm{b}}$ Scaling factors for each filter applied to the fractional stellar flux blocked by the planet to account for dilution from HAT-P-57B and HAT-P-57C in all of the light curves, and to account for over-filtering in the HATNet data.

${ }^{\mathrm{c}}$ Here $N(\mu, \sigma)$ corresponds to a normal distribution with mean $\mu$ and standard deviation $\sigma$. For the blend factors these are determined based on the measured magnitudes of HAT-P-57B and HAT-P-57C together with the PARSEC isochrones. For the line profile parameters these are determined from the posterior distributions for each parameter from the light curve and RV curve analysis.

${ }^{\mathrm{d}}$ Out-of-transit magnitude. One such parameter is used for each light curve in the analysis. For computational efficiency these parameters are optimized via linear least squares at each step in the MCMC.

${ }^{\mathrm{e}}$ EPD coefficients used to remove quadratic variations in time, or variations that are correlated with changes in the shape of the PSF. Five such parameters are used for each of the KeplerCam light curves. For computational efficiency these parameters are optimized via linear least squares at each step in the MCMC.

${ }^{\mathrm{f}}$ Center velocity of the line profile. One such parameter is used for each profile analyzed.

g Noise model parameters discussed in Section 3.3.

${ }^{\mathrm{h}}$ Parameter scaling the depth of the line profile. One such parameter is used for each profile analyzed.

${ }^{i}$ The continuum level of the line profile. One such parameter is used for each profile analyzed.
To model the broadening profile measurements we use an analytic expression for the rotational broadening kernel of a spherical star with quadratic limb-darkening, undergoing solid-body rotation and transited by a spherical nonluminous planet (see Appendix for the derivation). This fit was done separately from the modeling of the light curves and RVs discussed in Section 3.2, but to ensure that the constraints on the orbital parameters of the planet and its radius relative to the star are incorporated into the line profile fit, we used the posterior parameter distributions from the light curve and RV curve analysis to determine priors on these same parameters for the line profile fit. Our line profile model also depends on the projected angle between the spin axis of the host and the orbital axis of the planet $(\lambda)$, the maximum projected rotation velocity of the star $(v \sin i)$, the mean velocity of the star $(\gamma$, which we take to be a free parameter, and independent of the $\gamma$ velocity measured with other spectrographs or reductions of the Keck-I/HIRES data), the quadratic limb darkening coefficients $\left(u_{1}\right.$ and $\left.u_{2}\right)$ and two factors scaling and offsetting the model to match the measurements. For the limb darkening coefficients we vary the combinations $u_{1}^{\prime}$ and $u_{2}^{\prime}$ in the fit, with $u_{1}=0.576236 u_{1}^{\prime}+0.81732928 u_{2}^{\prime}$ and $u_{2}=-0.81732928 u_{1}^{\prime}+0.576236 u_{2}^{\prime}$, which we find to have uncorrelated posterior probability distributions, rather than varying $u_{1}$ and $u_{2}$ directly. The set of parameters that we vary in this fit, together with the adopted priors, are listed in Table 4.

As seen in Figure 10 the line profile residuals from the physical model exhibit correlated variations that are not associated with the planet. Based on inspecting the continuum region of the line profiles outside of the range shown in Figure 9 we find that the systematic variations are limited to within the line profile, so we conclude that the variations are most likely due to intrinsic stellar variability, evidence for which is also seen in the HATNet photometry (similar variations have also been seen in the line profile of WASP33, e.g., Collier Cameron et al. 2010b). In order to account for these systematic variations, which is especially important in determining accurate uncertainties for $\lambda$ and $v \sin i$, we use a non-diagonal covariance matrix in evaluating the likelihood function. We parameterize the covariance matrix using an exponential model, which we found by inspection to provide a good match to the autocorrelation of the residuals from the physical model. The covariance between points $i$ and $j$ with velocity differences $\Delta v_{i}$ and $\Delta v_{j}$ is taken to be:

$$
\Sigma_{i j}=\alpha \sigma_{i}^{2} \delta_{i j}+A \exp \left(-\left|\Delta v_{i}-\Delta v_{j}\right| / \rho\right)
$$

where $\sigma_{i}$ is our estimated uncertainty for point $i, \delta_{i j}$ is the Kronecker delta function, $\alpha$ is a parameter used to scale the uncertainties, and $A$ and $\rho$ are parameters describing the amplitude and length-scale for the covariance (i.e., we are using a Gaussian-process regression, or GP, to model the systematic variations, see Gibson et al. 2012 for a more detailed discussion of this technique). The likelihood of the data in column vector $y$ given the model in column vector $\boldsymbol{y}_{\text {mod }}$ parameterized by $\theta_{1}$ and with covariance matrix $\Sigma$ having parameters $\theta_{2}$ (i.e., $\alpha, A$ 
Table 5

Parameters for the Transiting Planet HAT-P-57 b

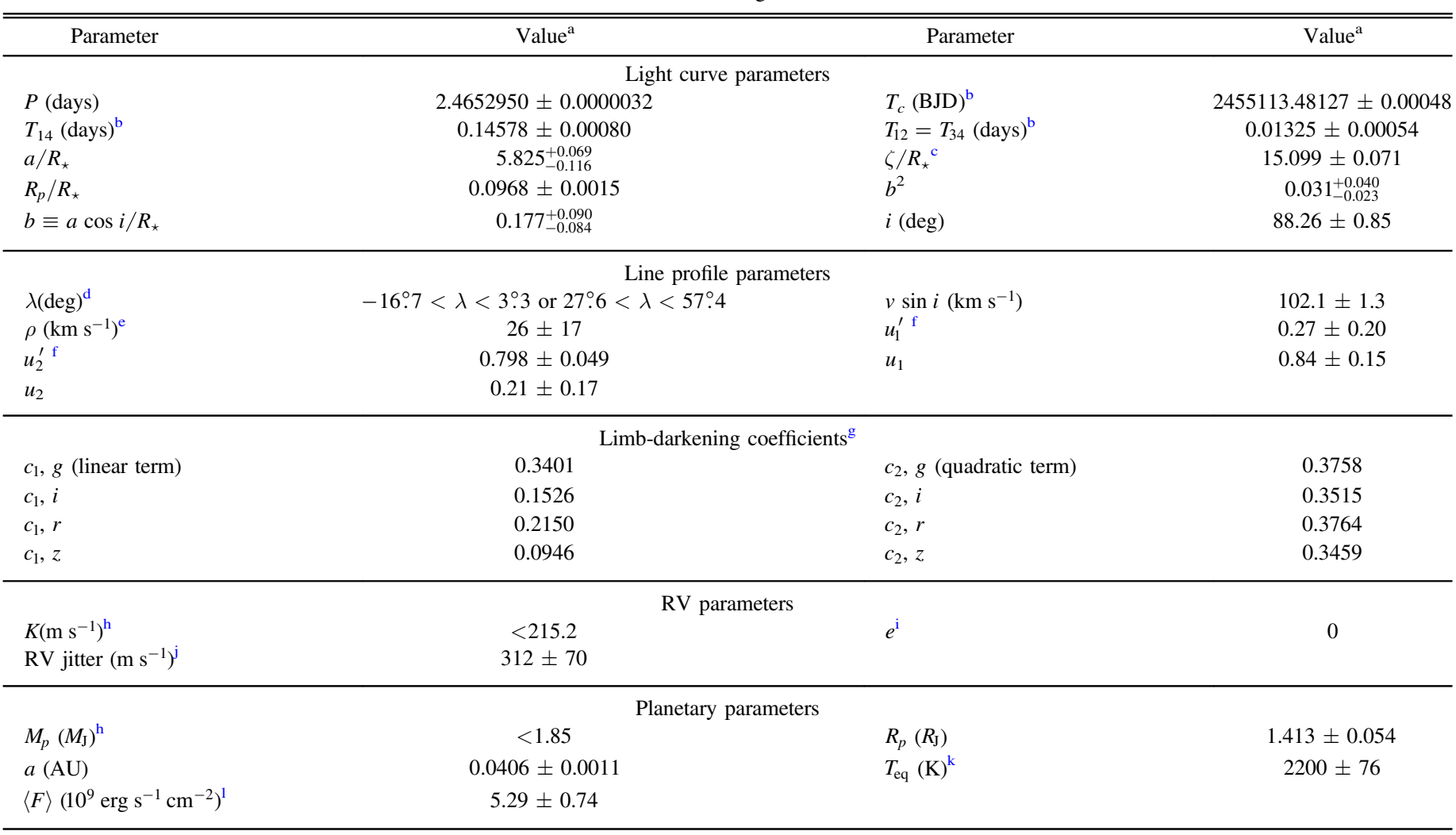

Notes.

${ }^{\text {a }}$ The adopted parameters assume a circular orbit. Based on the Bayesian evidence ratio we find that this model is strongly preferred over a model in which the eccentricity is allowed to vary in the fit. For each parameter we give the median value and $68.3 \%(1 \sigma)$ confidence intervals from the posterior distribution.

${ }^{\mathrm{b}}$ Reported times are in Barycentric Julian Date calculated directly from UTC, without correction for leap seconds. $T_{c}$ : reference epoch of mid transit that minimizes the correlation with the orbital period. $T_{14}$ : total transit duration, time between first to last contact; $T_{12}=T_{34}$ : ingress/egress time, time between first and second, or third and fourth contact.

${ }^{\mathrm{c}}$ Reciprocal of the half duration of the transit used as a jump parameter in our MCMC analysis in place of $a / R_{\star}$. It is related to $a / R_{\star}$ by the expression $\zeta / R_{\star}=a / R_{\star}(2 \pi(1+e \sin \omega)) /\left(P \sqrt{1-b^{2}} \sqrt{1-e^{2}}\right)($ Bakos et al. 2010).

${ }^{\mathrm{d}}$ The marginalized posterior probability distribution for $\lambda$ is multimodal. We list the ranges of $\lambda$ above the $95 \%$ confidence level. The two ranges have relative probabilities of $26 \%$ and $74 \%$, respectively.

e The exponential correlation length scale for describing systematic variations in the line profile (Equation (2)).

${ }^{\mathrm{f}}$ Uncorrelated quadratic limb darkening coefficients from modeling the spectral line profiles. These are related to the usual quadratic limb coefficients $u_{1}$ and $u_{2}$ via $u_{1}=0.576236 u_{1}^{\prime}+0.81732928 u_{2}^{\prime}$ and $u_{2}=-0.81732928 u_{1}^{\prime}+0.576236 u_{2}^{\prime}$.

g Values for a quadratic law, adopted from the tabulations by Claret (2004) according to the spectroscopic (SPC) parameters listed in Table 3.

h $95 \%$ confidence upper limit.

${ }^{i}$ We assume a circular orbit for the analysis.

${ }^{\mathrm{j}}$ Error term, either astrophysical or instrumental in origin, added in quadrature to the formal RV errors. This term is varied in the fit assuming a prior inversely proportional to the jitter.

${ }^{\mathrm{k}}$ Planet equilibrium temperature averaged over the orbit, calculated assuming a Bond albedo of zero, and that flux is reradiated from the full planet surface.

${ }^{1}$ Incoming flux per unit surface area, averaged over the orbit.

and $\rho$ ) is then

$$
\begin{aligned}
\log \mathcal{L}\left(\boldsymbol{y} \mid \theta_{1}, \theta_{2}\right)= & -\frac{1}{2}\left(\boldsymbol{y}-\boldsymbol{y}_{\text {mod, } \theta_{1}}\right)^{T} \boldsymbol{\Sigma}_{\boldsymbol{\theta}_{2}}^{-1}\left(\boldsymbol{y}-\boldsymbol{y}_{\text {mod, } \theta_{1}}\right) \\
& -\frac{1}{2} \log \left|\boldsymbol{\Sigma}_{\boldsymbol{\theta}_{2}}\right|+C
\end{aligned}
$$

for some constant $C$. We assume exponential priors for $\rho$ and $A$ and a Jeffreys prior for $\alpha$.

We run a DEMCMC analysis to explore this likelihood function for the line profiles, and determine the posterior distributions of the parameters. The maximum a posteriori model is shown in Figure 9. Figure 11 shows the marginalized posterior probability distribution for $\lambda$, while Figure 12 shows the correlations between $\lambda$ and $v \sin i$ and between $\lambda$ and $b$.

When the GP is used to model the systematic variations we find a multi-modal posterior distribution for $\lambda$, with the ranges $-10^{\circ} .9<\lambda<-4.1,34^{\circ} .2<\lambda<44^{\circ} .8$, and $47^{\circ} .3<\lambda<55^{\circ} .9$ having marginal posterior probability above the $68.3 \%$ confidence limit, and the ranges $-16.7<\lambda<3.3$ and $27^{\circ} .6<\lambda<57^{\circ} .4$ having marginal posterior probability above the $95 \%$ confidence limit. The relative probabilities of the two modes permitted at $95 \%$ confidence $\left(-16.7<\lambda<3.3\right.$, and $\left.27^{\circ} .6<\lambda<57^{\circ} .4\right)$ are $26 \%$ and $74 \%$, respectively. 

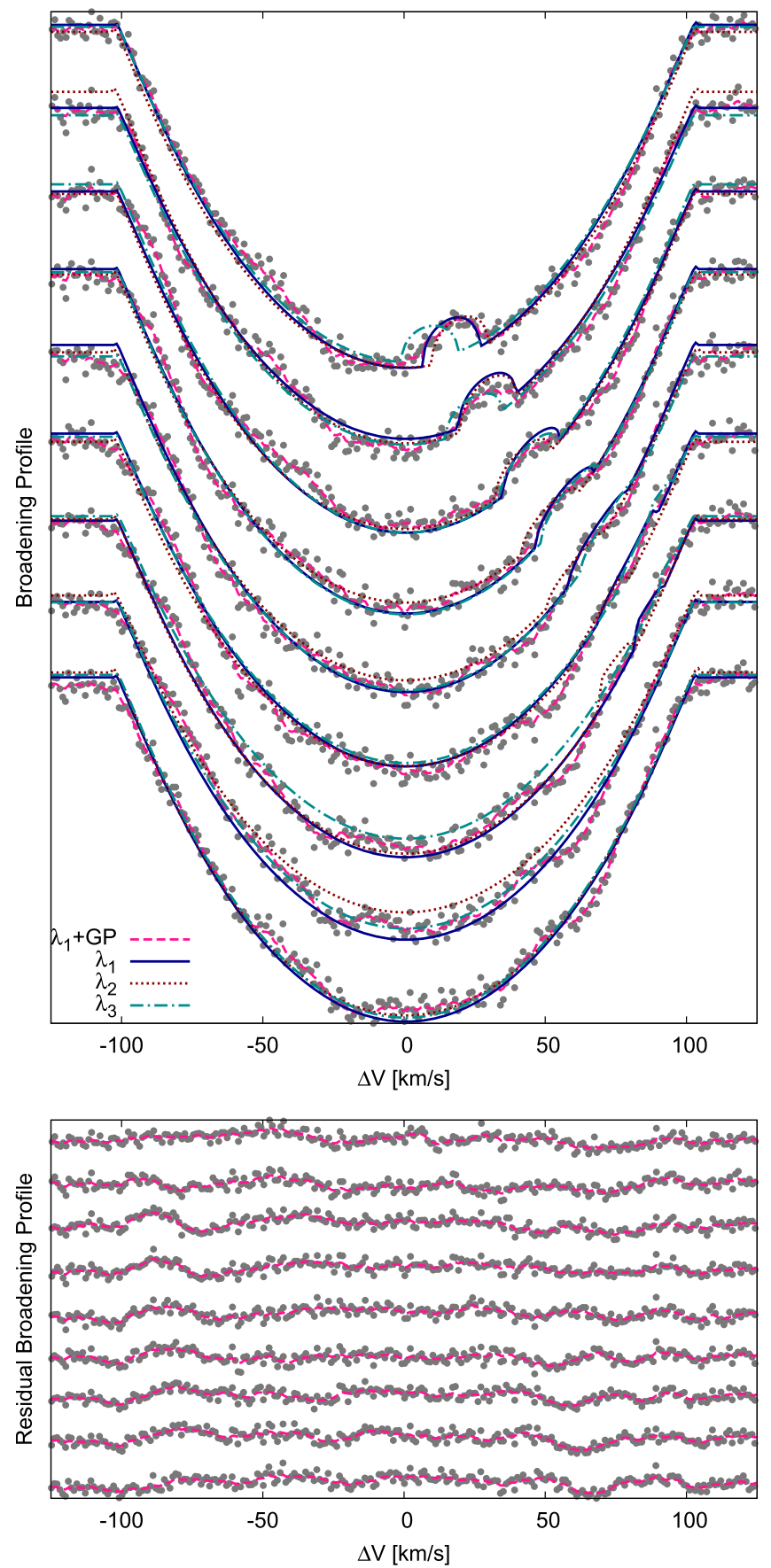

Figure 9. Top panel: the average rotational broadening profile of HAT-P-57 for nine consecutive Keck-I/HIRES observations proceeding chronologically from top to bottom. The first seven profiles are from observations obtained with HAT-P-57b in transit, while the bottom two are out of transit. The lines labeled $\lambda_{1}$ through $\lambda_{3}$ show models for a star undergoing solid body rotation with a quadratic limb darkening law and a TEP with $\lambda_{1}=37^{\circ} .6, \lambda_{2}=51^{\circ} .5$ and $\lambda_{3}=-7^{\circ} .7$ (in order from highest to lowest a posteriori probability, and corresponding to the three modes shown in Figure 11). The lines labeled $\lambda_{1}+G P$ shows the combination of the $\lambda_{1}=37^{\circ} .6$ physical model with a Gaussian Process Regression used to account for additional systematic variations in the data (see Section 3.3). The planet creates the bump in the profile seen at $\Delta V \sim 15 \mathrm{~km} \mathrm{~s}^{-1}$ in the first observation, and progressing to higher velocities in subsequent observations. Bottom panel: residuals from the $\lambda_{1}$ physical model with the Gaussian Process Regression overplotted on each. These are displayed in the same order as in the top panel.

The mode peaking at $\lambda=-7.7$ requires a relatively high impact parameter $(b \gtrsim 0.3$, Figure 12$)$, while the higher $\lambda$ modes require a lower impact parameter $(b \lesssim 0.2)$. This

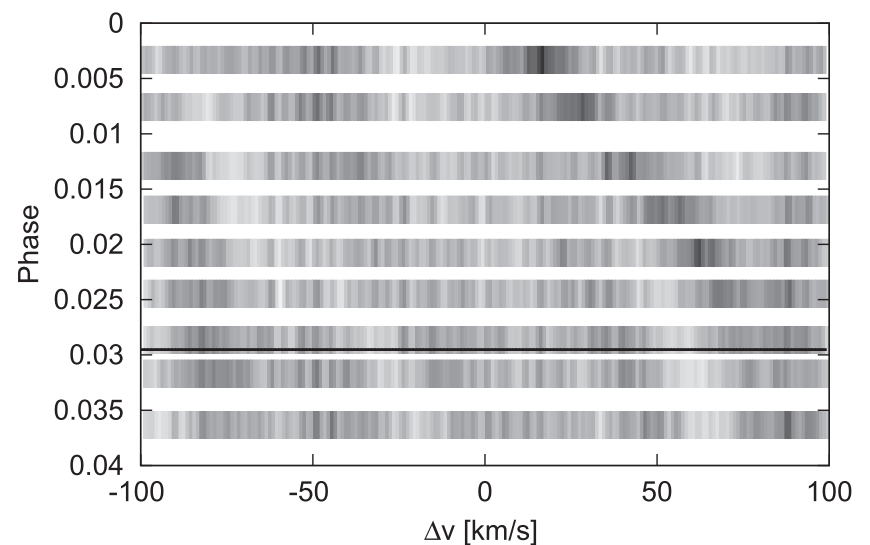

Figure 10. Residuals of the line profiles shown in Figure 9 from a simple model limb-darkened profile, without a planet, and without including the Gaussian Process. Zero phase corresponds to transit center, while the solid horizontal line marks the end of transit egress. The width of each band has been increased by a factor of 1.75 compared to the exposure time. The grayscale has been reversed such that dark areas correspond to positive residuals from the line profile model. The planet is seen as the darkest shadow moving diagonally downward and to the right.

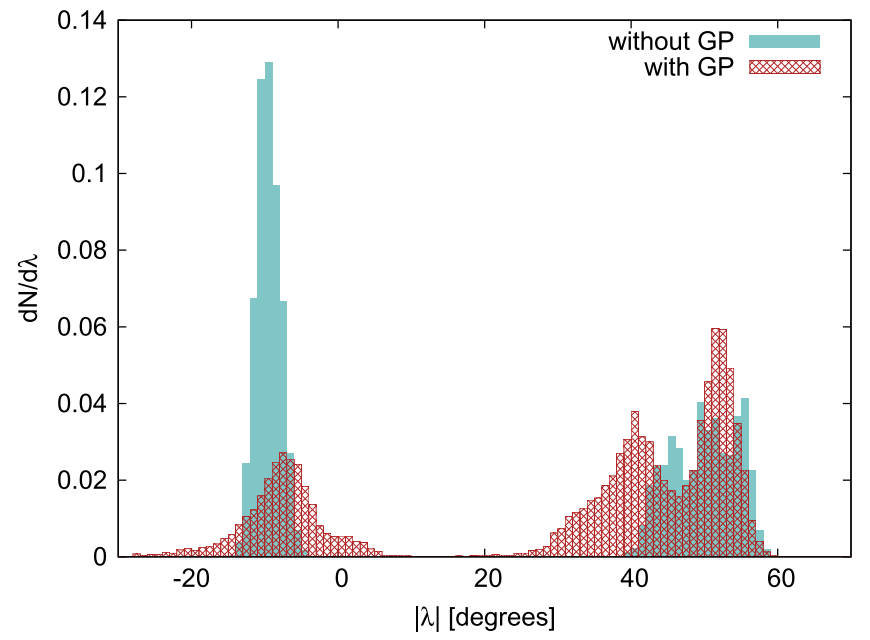

Figure 11. Posterior probability distribution for $\lambda$ based on modeling the line profiles (Figure 9). We compare the posterior distribution for the case when a Gaussian Process is included to account for additional systematic variations in the line profiles (histogram labeled "with GP") and for the case when a Gaussian Process is not used (histogram labeled "without GP"). The posterior distribution for $\lambda$ is multimodal.

degeneracy is due in part to the small number of line profile observations in which the planet shadow is clearly detected, and the lack of observations prior to transit center. Figure 9 shows both the overall maximum posterior probability model, which has $\lambda_{1}=37.6$ and the other two local maxima $\left(\lambda_{2}=51.5\right.$ and $\lambda_{3}=-7^{\circ} .7$ ), overplotted on the observed line profiles, while Figure 13 shows the projected geometry for these different orbital configurations. The models yield similar tracks for the planet in velocity space over the time-span of the observations. A longer time-base covering the full transit would allow the track of the planet to be determined, and not just its position in velocity space near transit center, helping to distinguish between these modes. Additionally, higher precision photometric follow-up to provide a tighter constraint on $b$ could also help break the degeneracy. Note, however, that as seen in Figure 13, based solely on Doppler tomography and 

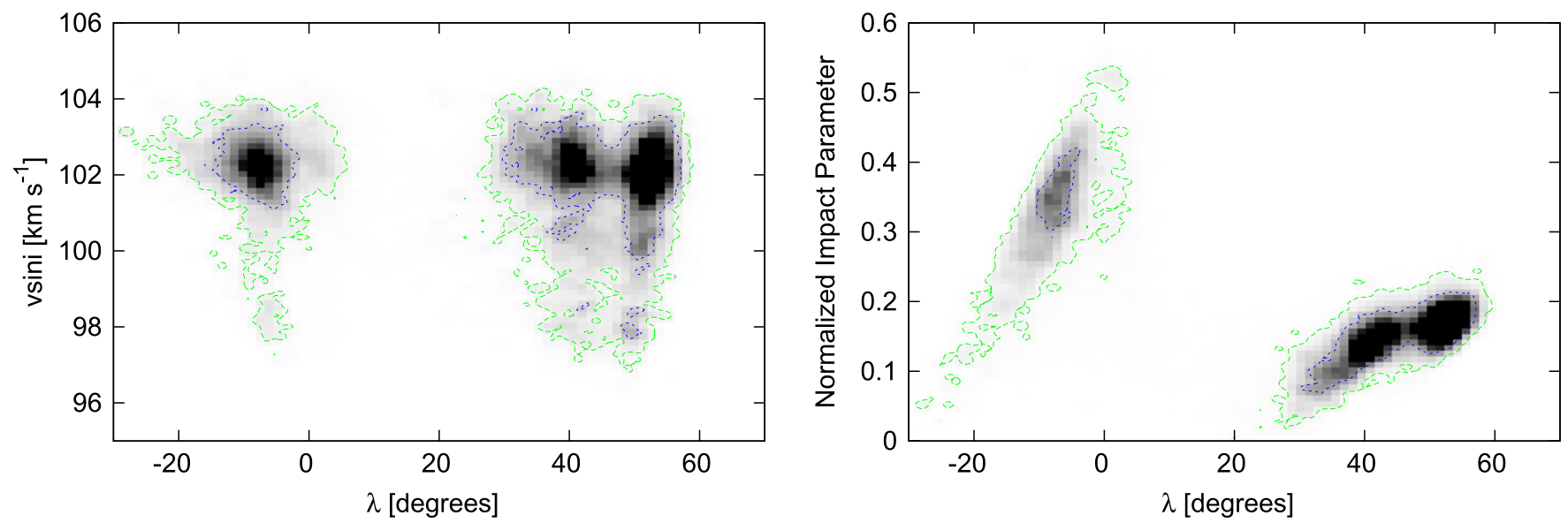

Figure 12. The marginalized joint posterior probability distributions for $v \sin i$ and $\lambda$ (left) and for $b$ and $\lambda$ (right) as determined from the Markov Chains produced in modeling the line profiles (Figure 9). The $68.3 \%$ and $95 \%$ confidence contours are overplotted with blue and green lines, respectively.

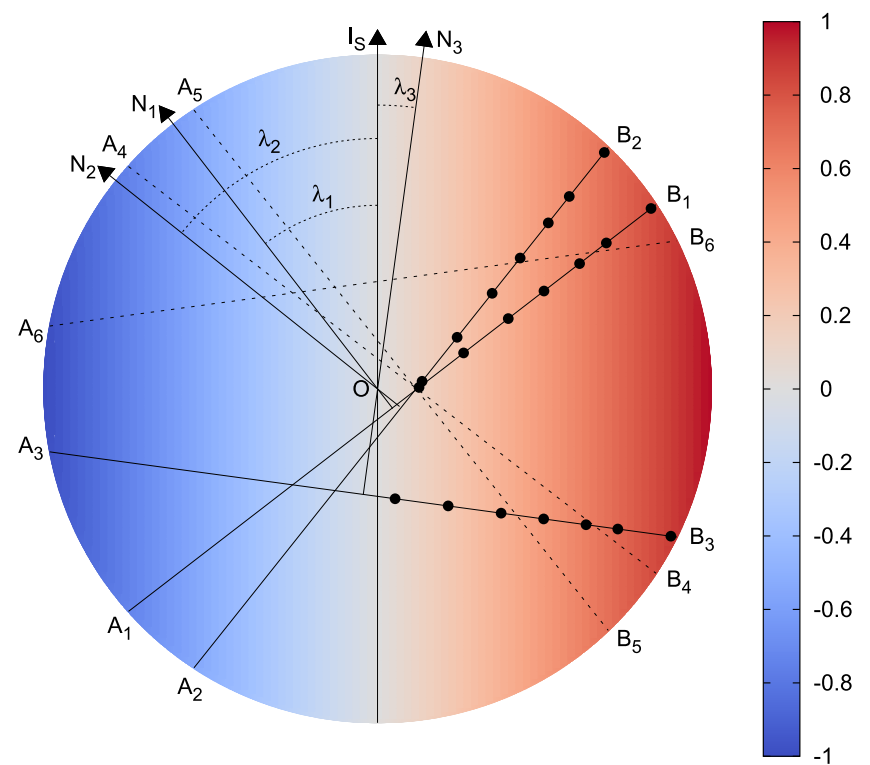

Figure 13. Geometry in the plane of the sky for orbital configurations of HATP-57b permitted by the line profile analysis. Vector $\boldsymbol{N}_{S}$ is the sky-projected spin axis of the star. The color shading indicates the rotational radial velocity at each point on the stellar surface. Transit chords $A_{1} B_{1}, A_{2} B_{2}$ and $A_{3} B_{3}$ are orbits with $\lambda_{1}=37^{\circ} .6, \lambda_{2}=51.5$, and $\lambda_{3}=-7.7$, respectively (corresponding to the three peaks in the $\lambda$ posterior distribution shown in Figure 11). These angles are measured between the spin axis of the star and the projected orbit normal vectors $N_{1}, N_{2}$, and $N_{3}$. Filled circles show the position of the planet at the times of the 7 Keck/HIRES observations obtained during transit. Tomography observations covering the first half of a transit would be able two distinguish between these three orbital configurations. We also show transit chords $A_{4} B_{4}$, $A_{5} B_{5}$ and $A_{6} B_{6}$ for which the tomography and light curve data are degenerate with $A_{1} B_{1}, A_{2} B_{2}$, and $A_{3} B_{3}$, respectively. These configurations have projected alignment angles of $180^{\circ}-\lambda$ and have the south pole of the orbit pointing toward the observer.

transit observations, orbits with projected alignment $\lambda$ and the north pole of the orbit pointing toward the observer are degenerate with orbits having projected alignment $180^{\circ}-\lambda$ and the south pole of the orbit pointing toward the observer (Fabrycky \& Winn 2009).

The distributions for $v \sin i$, the limb darkening coefficients, and the correlation length scale $\rho$ are all nearly Gaussian. In particular we find $v \sin i=102.1 \pm 1.3 \mathrm{~km} \mathrm{~s}^{-1}$ and $\rho=$ $26 \pm 17 \mathrm{~km} \mathrm{~s}^{-1}$.

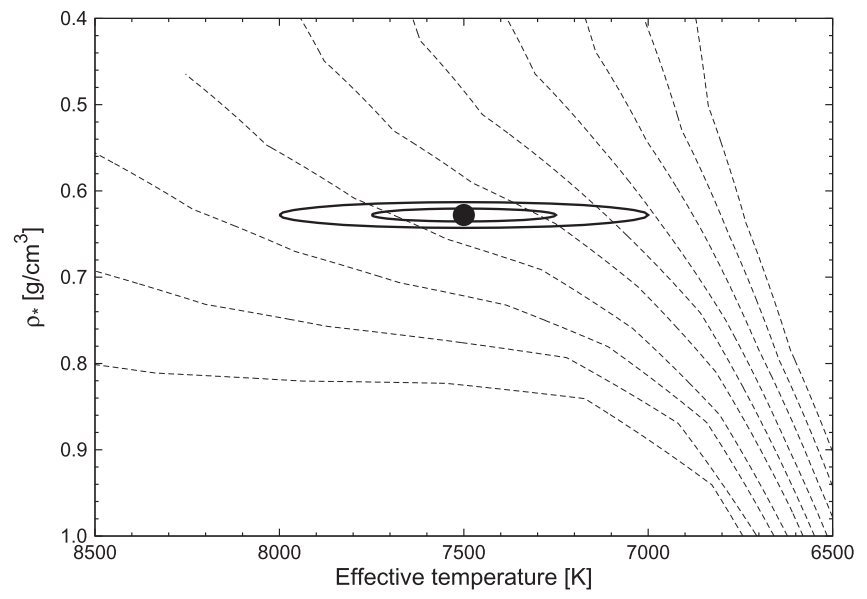

Figure 14. Comparison between the measured values of $T_{\text {eff } \star}$ and $\rho_{\star}$ (filled circle), and the $\mathrm{Y}^{2}$ model isochrones from Yi et al. (2001). The best-fit values, and approximate $1 \sigma$ and $2 \sigma$ confidence ellipsoids are shown. The $\mathrm{Y}^{2}$ isochrones are shown for ages of $0.2-2.0 \mathrm{Gyr}$, in $0.2 \mathrm{Gyr}$ increments.

For the limb darkening coefficients we find $u_{1}^{\prime}=$ $0.27 \pm 0.20$ and $u_{2}^{\prime}=0.798 \pm 0.049$, which correspond to $u_{1}=0.84 \pm 0.15$ and $u_{2}=0.21 \pm 0.17$. These differ significantly from the expected coefficients for the $g$-band from Claret (2004) for a star with the adopted atmospheric parameters of HAT-P-57. The expected values are $u_{1, g}=0.3401$ and $u_{2, g}=0.3758$, respectively. Practically speaking, the Claret (2004) coefficents predict a flatter profile in the center of the line and a steeper profile near the edge than what is observed. While errors in the model limb darkening coefficients have been noted based on, for example, Kepler transit observations (e.g., Espinoza \& Jordán 2015), the inferred $u_{1}$ coefficient based on our observations is much larger than other works have suggested. For example, for Kepler-13A, Müller et al. (2013) find $u_{1}=0.308 \pm 0.007$ and $u_{2}=0.222 \pm 0.014$ in the Kepler band-pass. While models predict $u_{1}$ to be higher in the bluer band-pass used in the Doppler tomography analysis, the expected difference is much less than what we measure. One possible explanation for the discrepancy is that a low order pulsation mode is distorting the overall line profile shape, leading to incorrect limb darkening estimates. To determine how systematic errors in the limb darkening affect our results, 
we have also carried out a fit with the quadratic limb darkening coefficients fixed to the Claret (2004) $g$-band values. We find that the posterior distribution for $\lambda$ is not significantly affected by the treatment of limb darkening. For $v \sin i$, on the other hand, we find that the value is more tightly constrained when the limb darkening coefficients are fixed, but that it is still consistent with the results when the coefficients are allowed to vary $\left(v \sin i=101.69 \pm 0.37 \mathrm{~km} \mathrm{~s}^{-1}\right.$ when the coefficients are fixed, compared to $v \sin i=102.1 \pm 1.3 \mathrm{~km} \mathrm{~s}^{-1}$ when they are allowed to vary).

For comparison, if we do not include the GP in the modeling, and instead assume zero covariance between points in the line spread function, then we find a bimodal, but much more tightly constrained, distribution for $\lambda$, with the ranges $-12^{\circ} .41<\lambda<-5^{\circ} .78$ and $40^{\circ} .89<\lambda<57^{\circ} .32$ having marginal posterior probability above the $68.3 \%$ confidence limit, and the ranges $-13.41<\lambda<-4.17$ and $39^{\circ} .80<\lambda<58^{\circ} .23$ having marginal posterior probability above the $95 \%$ confidence limit. In this case the two modes have relative probabilities of $55 \%$ and $45 \%$, respectively. The constraint on $v \sin i$ is also tighter with $v \sin i=101.46 \pm 0.19 \mathrm{~km} \mathrm{~s}^{-1}$.

Other methods for modeling the systematic variations in the line profiles were also considered. These include using a squared exponential kernel, and using a Fourier series, but we found that the former did not sufficiently account for long range correlations, while the latter had the undesirable effect of suppressing both the overall line shape and the planet signature. It is likely that a more physically motivated model for the systematics that accounts for the wavelike oscillations over the surface of the star, and their propagation in time, may provide a better description of the data, and reduce the uncertainty on $\lambda$ that results from suppressing the planet signature through overfitting with the GP. For example, the two-dimensional Fourier filtering technique used by Johnson et al. (2015) in their analysis of WASP-33 may work better at removing the stellar oscillations. The analysis in that case was facilitated by the retrograde motion of WASP-33, leading to a clean separation of the planet shadow and stellar oscillations in Fourier space. In the case of HAT-P-57 the planet is prograde with $\lambda$ close to zero, and thus difficult to separate from the stellar oscillations.

\subsection{Photometric Blend Analysis}

The detection of the Doppler shadow of HAT-P-57b moving across the rotational broadening profile of HAT-P-57 during a transit, and its consistency in amplitude and width with the $R_{p} / R_{\star}$ value measured from the transit light curves, provides confirmation that the transiting object is orbiting the bright rapidly rotating A star whose light dominates the spectrum. This, coupled with the upper limit of $K<215.2 \mathrm{~m} \mathrm{~s}^{-1}$ on the $\mathrm{RV}$ variation of this star, allows us to confirm that this is a TEP system, and not a blended stellar eclipsing binary system.

As an additional check on our conclusion that HAT-P-57 is not a blended stellar eclipsing binary system, we also carried out a blend analysis following Hartman et al. (2012). We find that, based on the photometry alone, all blended stellar eclipsing binary models that we tested provide a fit to the data that has a higher $\chi^{2}$ than the best-fit star+planet model. All of these blend models can be rejected with greater than $5 \sigma$ confidence in favor of the star+planet model. Moreover, based on the MMT/Clio2 imaging, any unaccounted-for blending companion bright enough to influence the derived parameters of the system must be within $\sim 0$ ". 25 of HAT-P-57 (Figure 6).

\section{DISCUSSION}

In this paper we have presented the discovery of HAT-P$57 \mathrm{~b}$, a short-period $(P=2.4653$ days $)$ giant planet transiting a rapidly rotating $\mathrm{A} 8 \mathrm{~V}$ star. Periodic photometric transits in the light curve of this source have been independently detected by three separate transit surveys (HAT, WASP and KELT; see Section 2.1.1). Here we combine the HATNet photometry, with follow-up photometry from FLWO $1.2 \mathrm{~m} /$ KeplerCam and spectroscopy from Keck-I/HIRES, NOT/FIES and FLWO $1.5 \mathrm{~m} /$ TRES to confirm that this is a transiting planet system and to determine its properties. The confirmation follows from three pieces of observational evidence: (1) KeckI/HIRES spectroscopy obtained during a transit reveals the Doppler shadow of the planet HAT-P-57b moving across the average spectral absorption line profile of the star HAT-P-57. The consistency of the shape of the shadow with the transiting planet parameters measured from the light curve proves that the transiting object is not orbiting a fainter object blended by the bright A star whose light dominates the spectrum. (2) Keck-I/ HIRES RVs obtained out of transit allow us to place an upper limit on the semiamplitude of the RV orbital variation of the A star of $K<215.2 \mathrm{~m} \mathrm{~s}^{-1}$, and a corresponding upper limit on the mass of the transiting object of $M<1.85 M_{\mathrm{J}}$. (3) A blend analysis of the available photometric data rules out blended eclipsing binary scenarios in favor of a single star with a transiting planet with greater than $5 \sigma$ confidence.

Based on our analysis of the photometric and spectroscopic data, together with the $\mathrm{Y}^{2}$ stellar evolution models, we conclude that the star HAT-P-57 has a mass of $1.47 \pm 0.12 M_{\odot}$, a radius of $1.500 \pm 0.050 R_{\odot}$, and is located at a distance of $303 \pm 13 \mathrm{pc}$ from the Solar System. The planet HAT-P-57b has a radius of $1.413 \pm 0.054 R_{\mathrm{J}}$, a semimajor axis of $0.0406 \pm 0.0011 \mathrm{AU}$, and an estimated equilibrium temperature (assuming zero albedo and complete redistribution of heat) of $2200 \pm 76 \mathrm{~K}$.

AO imaging in $H$ and $L^{\prime}$ bands performed with MMT/Clio2 reveals a pair of stars separated 2!. 7 from HAT-P-57 and 0.! 22 from each other. The stars have $H$ and $L^{\prime}$ magnitudes consistent with being stars of mass $0.61 \pm 0.10 M_{\odot}$ and $0.53 \pm 0.08 M_{\odot}$ located at the same distance from the Solar System as HAT-P57. If they are physically associated with HAT-P-57, then this is a hierarchical triple star system with HAT-P-57B and HAT$\mathrm{P}-57 \mathrm{C}$ having a projected physical separation of $68 \pm 3 \mathrm{AU}$, and approximate orbital period of 500 year (assuming the projected separation corresponds to the physical semimajor axis of the orbit), while the HAT-P-57B +HAT-P-57C binary has a projected physical separation of $800 \pm 30$ AU from HAT-P-57 and an approximate orbital period of 14,000 year.

There are two factors which distinguish HAT-P-57b from the more than 1200 other confirmed or validated transiting planet systems. With a projected equatorial rotation velocity of $v \sin i=102.1 \pm 1.3 \mathrm{~km} \mathrm{~s}^{-1}$, HAT-P-57 has the highest rotation velocity of any star known to host a transiting planet. The next most rapidly rotating stars with transiting planets are KOI$89\left(v \sin i \approx 90 \mathrm{~km} \mathrm{~s}^{-1}\right.$, Ahlers et al. 2015), WASP-33 ( $v \sin i=86.48 \pm 0.06 \mathrm{~km} \mathrm{~s}^{-1}$, Collier Cameron et al. 2010b), Kepler-13A ( $v \sin i=76.6 \pm 0.2 \mathrm{~km} \mathrm{~s}^{-1}$, Santerne et al. 2012), and KELT-7 ( $v \sin i=65 \pm 6 \mathrm{~km} \mathrm{~s}^{-1}$, Bieryla et al. 2015). HAT-P-57, together with WASP-33, Kepler-13A, 
and KOI-89, are also the only four stars of spectral type earlier than F0 known to host transiting planets. This makes HAT-P$57 \mathrm{~b}$ a valuable system for studying the properties of close-in planets around rapidly rotating, relatively high mass stars.

While the rapid rotation of HAT-P-57 prevents us from measuring the mass of the planet through the RV orbital wobble of the host star, it also creates an opportunity to characterize the orbital geometry of the planetary system with unusually high accuracy. We have already taken initial steps in this direction through our modeling of the spectral line profiles during a partial transit event observed with Keck-I/HIRES. We constrain the projected angle between the spin axis of the host star and the orbital axis of the planet to lie within the range $-16.7<\lambda<3.3$ or $27^{\circ} .6<\lambda<57^{\circ} .4$ with $95 \%$ confidence. These two distinct modes have relative probabilities of $26 \%$ and $74 \%$, respectively. While we do not find a unique solution, we are able to rule out very high obliquities, and conclude that HAT-P$57 \mathrm{~b}$ is either moderately misaligned in projection (most likely) or it is close to being aligned in projection (less likely, but still possible). Additional Doppler tomography observations, especially observations covering a full transit, will be able to distinguish between these two scenarios and pin down the angle $\lambda$.

If HAT-P-57b is not on a well-aligned orbit, then the significant expected oblateness for HAT-P-57, resulting from its rapid rotation, should cause the orbit of HAT-P-57b to precess at a relatively rapid rate. This would be observable by measuring changes in $\lambda$ and/or $b$ over time, as has been done for WASP-33b (Johnson et al. 2015) and Kepler-13Ab (Szabó et al. 2012; Masuda 2015). Measuring the precession rate would provide an observational constraint on the $J_{2}$ gravitational quadrupole moment of the star, which in turn may lead to a better age determination for the system, and/or can be used to test the theoretical stellar evolution models themselves.

The rapid rotation of HAT-P-57 also makes possible a measurement of the true (not projected) spin-orbit alignment angle by detecting an asymmetry in the transit shape resulting from gravity darkening in the oblate star (Masuda 2015) (the degeneracy between orbits having projected alignment angle $180^{\circ}-\lambda$ seen in Figure 13 may also be lifted by this technique). Following van Belle et al. (2004), we estimate that HAT-P-57 has an oblateness of $R_{b} / R_{a} \approx 1.03$, where $R_{b}$ and $R_{a}$ are the equatorial and polar radii of the star, respectively. This is quite similar to the estimate for WASP-33. Following Zhou \& Huang (2013), we estimate that the maximum difference between transit models including and excluding the gravity darkening effect is $\approx 500 \mathrm{ppm}$, assuming the rotation axis of the star lies in the plane of the sky. Detecting such a signal may be possible, though challenging, from the ground. It should also be detectable by TESS (based on its position on the sky, we expect TESS to monitor HAT-P-57 for $\sim 27$ days). This will depend, however, on whether HAT-P-57 exhibits high frequency (i.e., $\delta$-Scuti or roAp-type variations) photometric oscillations with amplitudes greater than the gravity darkening effect. While the HATNet light curve rules out high frequency oscillations with an amplitude above $1.2 \mathrm{mmag}$ in $r$-band (the observed low frequency oscillations are on a long enough time scale that it should be possible to filter these from transit light curves), the variations seen in the Keck-I/HIRES line profiles indicate that lower amplitude high frequency oscillations may be present.
We are grateful to the anonymous referee for their careful review of this paper, including several important comments. HATNet operations have been funded by NASA grants NNG04GN74G and NNX13AJ15G. Follow-up of HATNet targets has been partially supported through NSF grant AST1108686. G. Á. B., Z. C. and K. P. acknowledge partial support from NASA grant NNX09AB29G. J. H. acknowledges support from NASA grant NNX14AE87G. K.P. acknowledges support from NASA grant NNX13AQ62G. We acknowledge partial support also from the Kepler Mission under NASA Cooperative Agreement NCC2-1390 (D. W. L., PI). We would also like to thank J. Pepper for informing us about the KELT observations of this system. Data presented in this paper are based on observations obtained at the HAT station at the Submillimeter Array of SAO, and the HAT station at the Fred Lawrence Whipple Observatory of SAO. The authors wish to recognize and acknowledge the very significant cultural role and reverence that the summit of Mauna Kea has always had within the indigenous Hawaiian community. We are most fortunate to have the opportunity to conduct observations from this mountain. This paper presents observations made with the Nordic Optical Telescope, operated on the island of La Palma jointly by Denmark, Finland, Iceland, Norway, and Sweden, in the Spanish Observatorio del Roque de los Muchachos of the Instituto de Astrofísica de Canarias.

\section{APPENDIX \\ ANALYTIC ROTATIONAL BROADENING KERNEL FOR A QUADRATICALLY LIMB DARKENED STAR WITH A TRANSITING PLANET}

The Doppler tomography method has been used to determine the spin-orbit alignments of the HD 189733, HAT-P-2, WASP32, WASP-33, WASP-38, Kepler-13A, Kepler-25, and KOI-12 transiting planet systems (Collier Cameron et al. 2010a, 2010b; Brown et al. 2012; Albrecht et al. 2013; Johnson et al. 2014b, 2015; Bourrier et al. 2015). These previous applications have modeled the rotational broadening function by using an analytic model for the broadening profile of a star with linear limb darkening and a transiting planet having $R_{P} / R_{\star} \ll 1$ (i.e., the planet shadow is treated as a Gaussian, Collier Cameron et al. 2010a, 2010b; Brown et al. 2012; Bourrier et al. 2015), or by carrying out a numerical integration of a gridded stellar surface brightness profile (Albrecht et al. 2013; Johnson et al. 2014b, 2015). The rotational broadening function for a star with linear limb darkening, undergoing solid body rotation, and being eclipsed by another object has a simple analytic form which was worked out by Kopal (1959, p. 208). Here we provide the analogous formula for a quadratic limb darkening law. We caution that this relation is only applicable for large rotation rates where macro-turbulence may be neglected.

The spectrum of a rotating star can be calculated by convolving the non-rotating spectrum with a broadening kernel (e.g., Gray 2005), i.e.,

$$
S_{R}(\lambda)=\int_{-\infty}^{\infty} S\left(\lambda\left(1-\tilde{v} v_{L} / c\right)\right) G(\tilde{v}) d \tilde{v}
$$

where $S(\lambda)$ is the non-rotating spectrum at wavelength $\lambda, v_{L}$ is the projected rotation velocity of the star, $c$ is the speed of light, and $G(\tilde{v})$ is the broadening kernel evaluated at relative velocity shift $\tilde{v}$. Following Gray (2005), the rotational broadening 
kernel is given by:

$$
G(\tilde{v})=\frac{G^{\prime}(\tilde{v})}{\int_{-\infty}^{\infty} G^{\prime}\left(v^{\prime}\right) d v^{\prime}}
$$

with

$$
G^{\prime}(\tilde{v})=\int_{y_{\min }(\tilde{v})}^{y_{\max }(\tilde{v})} I(\tilde{v}, y) d y
$$

being the integral of the stellar surface brightness $I(x, y)$ along a line of constant $x=\tilde{v}$. Here we are using a coordinate system centered on the stellar disk with the $y$ axis parallel to the projected rotation axis of the star, and with $x$ and $y$ measured in units of the stellar radius (note that for solid body rotation the projected rotation velocity is constant along a line of constant $x$ ). We also have $y_{\min }(x)=-\sqrt{1-x^{2}}$ and $y_{\max }(x)=\sqrt{1-x^{2}}$.

For a quadratic limb darkening law of the form

$$
I(\theta)=I_{c}\left(1-u_{1}(1-\cos (\theta))-u_{2}(1-\cos (\theta))^{2}\right)
$$

with $\theta$ being the angle between the line normal to the stellar surface and the line of sight from the center of the star to the observer, Equation (6) works out to

$$
\begin{aligned}
& G^{\prime}(\tilde{v}) \\
& = \begin{cases}2\left(1-u_{1}-u_{2}\right) \sqrt{1-\tilde{v}^{2}} \\
+\frac{\pi}{2}\left(u_{1}+2 u_{2}\right)\left(1-\tilde{v}^{2}\right)-\frac{4}{3} u_{2}\left(1-\tilde{v}^{2}\right)^{3 / 2} & |\tilde{v}|<1 \\
0 & |\tilde{v}| \geqslant 1\end{cases}
\end{aligned}
$$

and

$$
\int_{-\infty}^{\infty} G^{\prime}\left(v^{\prime}\right) d v^{\prime}=\pi\left(1-u_{1} / 3-u_{2} / 6\right)
$$

When a transiting planet with radius $R$ (in units of the stellar radius), is in front of the star at projected position $\left(x_{P}, y_{P}\right)$ relative to the center of the star (these are determined from the orbital parameters and the projected spin-orbit angle $\lambda$ following, e.g., Boué et al. 2013), we must subtract

$$
K(\tilde{v})=\int_{y_{1}\left(\tilde{v}, x_{P}, y_{P}, R\right)}^{y_{2}\left(\tilde{v}, x_{P}, y_{P}, R\right)} I(\tilde{v}, y) d y,
$$

the integral of the stellar surface brightness blocked by the planet, from Equation (8). For a quadratic limb darkening law we have

$$
\begin{aligned}
K(\tilde{v})= & \left(y_{2}-y_{1}\right)\left(1-u_{1}-u_{2}\left(2-\tilde{v}^{2}\right)\right) \\
& +\left(\frac{1}{2} u_{1}+\frac{2}{3} u_{2}\right)\left(y_{1}^{3}-y_{2}^{3}\right) \\
& +\frac{1}{2}\left(u_{1}+2 u_{2}\right)\left[\left(y_{2}-y_{1}\right)\left(1-\tilde{v}^{2}\right)+\left(1-\tilde{v}^{2}\right)\right. \\
& \left.\times\left(\sin ^{-1}\left(\frac{y_{2}}{\sqrt{1-\tilde{v}^{2}}}\right)-\sin ^{-1}\left(\frac{y_{1}}{\sqrt{1-\tilde{v}^{2}}}\right)\right)\right]
\end{aligned}
$$

with

$$
\begin{aligned}
& y_{1}\left(\tilde{v}, x_{P}, y_{P}, R\right)= \\
& \begin{cases}0 & |\tilde{v}| \geqslant 1 \text { or }\left|\tilde{v}-x_{P}\right| \geqslant R \\
\sqrt{1-\tilde{v}^{2}} & y_{P}-\sqrt{R^{2}-\left(\tilde{v}-x_{P}\right)^{2}} \geqslant \sqrt{1-\tilde{v}^{2}} \\
-\sqrt{1-\tilde{v}^{2}} & y_{P}-\sqrt{R^{2}-\left(\tilde{v}-x_{P}\right)^{2}} \leqslant \sqrt{1-\tilde{v}^{2}} \\
y_{P}-\sqrt{R^{2}-\left(\tilde{v}-x_{P}\right)^{2}} & \text { otherwise }\end{cases}
\end{aligned}
$$

and

$$
\begin{aligned}
& y_{2}\left(\tilde{v}, x_{P}, y_{P}, R\right)= \\
& \begin{cases}0 & |\tilde{v}| \geqslant 1 \text { or }\left|\tilde{v}-x_{P}\right| \geqslant R \\
\sqrt{1-\tilde{v}^{2}} & y_{P}+\sqrt{R^{2}-\left(\tilde{v}-x_{P}\right)^{2}} \geqslant \sqrt{1-\tilde{v}^{2}} \\
-\sqrt{1-\tilde{v}^{2}} & y_{P}+\sqrt{R^{2}-\left(\tilde{v}-x_{P}\right)^{2}} \leqslant \sqrt{1-\tilde{v}^{2}} \\
y_{P}+\sqrt{R^{2}-\left(\tilde{v}-x_{P}\right)^{2}} & \text { otherwise. }\end{cases}
\end{aligned}
$$

Examples of this model fit to the measured broadening profiles of HAT-P-57 are shown in Figure 9, where we have inverted the model profile to look like absorption lines for display purposes.

\section{REFERENCES}

Ahlers, J. P., Barnes, J. W., \& Barnes, R. 2015, arXiv:1509.07930 Albrecht, S., Winn, J. N., Marcy, G. W., et al. 2013, ApJ, 771, 11 Baines, E. K., White, R. J., Huber, D., et al. 2012, ApJ, 761, 57 Bakos, G., Noyes, R. W., Kovács, G., et al. 2004, PASP, 116, 266 Bakos, G. Á, Torres, G., Pál, A., et al. 2010, ApJ, 710, 1724 Bieryla, A., Hartman, J. D., Bakos, G. Á, et al. 2014, AJ, 147, 84 Bieryla, A., Collins, K., Beatty, T. G., et al. 2015, AJ, 150, 12 Biller, B. A., Males, J., Rodigas, T., et al. 2014, ApJL, 792, L22

Boué, G., Montalto, M., Boisse, I., Oshagh, M., \& Santos, N. C. 2013, A\&A, 550, A53

Bourrier, V., Lecavelier des Etangs, A., Hébrard, G., et al. 2015, A\&A, 579, A55

Bowler, B. P., Johnson, J. A., Marcy, G. W., et al. 2010, ApJ, 709, 396

Bressan, A., Marigo, P., Girardi, L., et al. 2012, MNRAS, 427, 127

Brown, D. J. A., Collier Cameron, A., Diaz, R. F., et al. 2012, ApJ, 760, 139

Buchhave, L. A., Bakos, G. Á, Hartman, J. D., et al. 2010, ApJ, 720, 1118

Buchhave, L. A., Latham, D. W., Johansen, A., et al. 2012, Natur, 486, 375

Butler, R. P., Marcy, G. W., Williams, E., et al. 1996, PASP, 108, 500

Cardelli, J. A., Clayton, G. C., \& Mathis, J. S. 1989, ApJ, 345, 245

Carson, J., Thalmann, C., Janson, M., et al. 2013, ApJL, 763, L32

Claret, A. 2004, A\&A, 428, 1001

Collier Cameron, A., Bruce, V. A., Miller, G. R. M., Triaud, A. H. M. J., \& Queloz, D. 2010a, MNRAS, 403, 151

Collier Cameron, A., Wilson, D. M., West, R. G., et al. 2007, MNRAS, 380,1230

Collier Cameron, A., Guenther, E., Smalley, B., et al. 2010b, MNRAS, 407, 507

Donati, J.-F., Semel, M., Carter, B. D., Rees, D. E., \& Collier Cameron, A. 1997, MNRAS, 291, 658

Espinoza, N., \& Jordán, A. 2015, MNRAS, 450, 1879

Fabrycky, D. C., \& Winn, J. N. 2009, ApJ, 696, 1230

Füresz, G. 2008, PhD thesis, Univ. Szeged

Freed, M., Hinz, P. M., Meyer, M. R., Milton, N. M., \& Lloyd-Hart, M. 2004 Proc. SPIE, 5492, 1561

Gibson, N. P., Aigrain, S., Roberts, S., et al. 2012, MNRAS, 419, 2683

Gray, D. F. 2005, The Observation and Analysis of Stellar Photospheres (Cambridge: Cambridge Univ. Press)

Gustafsson, B., Edvardsson, B., Eriksson, K., et al. 2008, A\&A, 486, 951

Handler, G., \& Shobbrook, R. R. 2002, MNRAS, 333, 251

Hartman, J. D., Bakos, G. Á, Béky, B., et al. 2012, AJ, 144, 139

Hartman, J. D., Bakos, G. Á, Torres, G., et al. 2014, AJ, 147, 128

Hinkley, S., Kraus, A. L., Ireland, M. J., et al. 2015, ApJL, 806, L9 
Johnson, J. A., Clanton, C., Howard, A. W., et al. 2011, ApJS, 197, 26 Johnson, J. A., Morton, T. D., \& Wright, J. T. 2013, ApJ, 763, 53

Johnson, J. A., Huber, D., Boyajian, T., et al. 2014a, ApJ, 794, 15

Johnson, M. C., Cochran, W. D., Albrecht, S., et al. 2014b, ApJ, 790, 30

Johnson, M. C., Cochran, W. D., Collier Cameron, A., \& Bayliss, D. 2015, arXiv: 1508.02398

Kopal, Z. 1959, Close Binary Systems (London: Chapman and Hall)

Kovács, G., Bakos, G., \& Noyes, R. W. 2005, MNRAS, 356, 557

Kovács, G., Zucker, S., \& Mazeh, T. 2002, A\&A, 391, 369

Kovács, G., Kovács, T., Hartman, J. D., et al. 2013, A\&A, 553, A44

Lafrenière, D., Jayawardhana, R., Janson, M., et al. 2011, ApJ, 730, 42

Lagrange, A.-M., Gratadour, D., Chauvin, G., et al. 2009, A\&A, 493, L21

Lehmann, H., Guenther, E., Sebastian, D., et al. 2015, A\&A, 578, L4

Lister, T. A., West, R. G., Wilson, D. M., et al. 2007, MNRAS, 379, 647

Lloyd, J. P. 2011, ApJL, 739, L49

Lloyd, J. P. 2013, ApJL, 774, L2

Mandel, K., \& Agol, E. 2002, ApJL, 580, L171

Marcy, G. W., \& Butler, R. P. 1992, PASP, 104, 270

Marois, C., Macintosh, B., Barman, T., et al. 2008, Sci, 322, 1348

Masuda, K. 2015, ApJ, 805, 28

Mazeh, T., Nachmani, G., Sokol, G., Faigler, S., \& Zucker, S. 2012, A\&A, 541, A56

Müller, H. M., Huber, K. F., Czesla, S., Wolter, U., \& Schmitt, J. H. M. M. 2013, A\&A, 560, A112

Palacios, A., Gebran, M., Josselin, E., et al. 2010, A\&A, 516, A13
Pecaut, M. J., \& Mamajek, E. E. 2013, ApJS, 208, 9

Queloz, D., Henry, G. W., Sivan, J. P., et al. 2001, A\&A, 379, 279

Rodríguez, E., \& Breger, M. 2001, A\&A, 366, 178

Rowe, J., Borucki, W. J., Howell, S. B., et al. 2011, in American Astronomical Society Meeting Abstracts, 217, 103.04

Rowe, J. F., Bryson, S. T., Marcy, G. W., et al. 2014, ApJ, 784, 45

Royer, F., Zorec, J., \& Gómez, A. E. 2007, A\&A, 463, 671

Santerne, A., Moutou, C., Barros, S. C. C., et al. 2012, A\&A, 544, L12

Sato, B., Omiya, M., Harakawa, H., et al. 2012, PASJ, 64, 135

Schlaufman, K. C., \& Winn, J. N. 2013, ApJ, 772, 143

Shporer, A., Jenkins, J. M., Rowe, J. F., et al. 2011, AJ, 142, 195

Siverd, R. J., Beatty, T. G., Pepper, J., et al. 2012, ApJ, 761, 123

Spiegel, D. S., \& Burrows, A. 2012, ApJ, 745, 174

Szabó, G. M., Pál, A., Derekas, A., et al. 2012, MNRAS, 421, L122

Telting, J. H., Avila, G., Buchhave, L., et al. 2014, AN, 335, 41

ter Braak, C. J. F. 2006, Statistics and Computing, 16, 239

Torres, G., Bakos, G. Á, Kovács, G., et al. 2007, ApJL, 666, L121

van Belle, G. T., Ciardi, D. R., Thompson, R. R., \& Akeson, R. L. 2004, in

IAU Symp. 215, Stellar Rotation, ed. A. Maeder \& P. Eenens (San Francisco, CA: ASP), 177

Vogt, S. S., Allen, S. L., Bigelow, B. C., et al. 1994, Proc. SPIE, 2198, 362

Wittenmyer, R. A., Endl, M., Wang, L., et al. 2011, ApJ, 743, 184

Yi, S., Demarque, P., Kim, Y.-C., et al. 2001, ApJS, 136, 417

Zechmeister, M., \& Kürster, M. 2009, A\&A, 496, 577

Zhou, G., \& Huang, C. X. 2013, ApJL, 776, L35 